\title{
On the theories and numerics of continuum models for adaptation processes in biological tissues
}

P. Saez

Received: XX.XX.XX/ Revised version: XX.XX.XX

\begin{abstract}
Computational continuum mechanics have been used for a long time to deal with the mechanics of materials. During the last decades researches have been using many of the theoretical models and numerical approaches of classical materials to deal with biological tissue which, in many senses, are a much more sophisticated material. We aim to review the last achievements of continuum models and numerical approaches on adaptation processes in biological tissues. In this review, we are looking, in particular, at growth in terms of changes of density and/or volume as, e.g., in collagen remodeling, wound healing, arterial thickening, etc. Furthermore, we point out some of the most relevant limitations of the current state-of-the-art in terms of these well established computational continuum models. In connection with these limitations, we will finish by discussing the trend lines of future work in the field of modeling biological adaptation, focusing on the computational approaches and mechanics that could overcome the current drawbacks. We would also like to attract the attention of all those researchers in classical materials (metal, alloys, composites, etc), to point out how similar the continuum and computational models between our fields are. We hope we can motivate them for getting their expertize in this challenging field of research.
\end{abstract}

Keywords Open systems · Continuum mechanics · Growth · Remodeling · FEM

Mathematical Institute, University of Oxford, Oxford, UK

E-mail: saez@maths.ox.ac.uk 


\section{Contents}

\section{Motivation}

Nowadays, the mathematical and computational study of biomechanics and biology have gained a huge significance in the research community. There have been an important increase in the number of scientific forums devote to them. While some years or decades ago bioengineering works were doomed to be presented in general continuum mechanics and computational journals.

The study of plasticity, damage, viscoelasticity, etc., have been key points of development in continuum mechanics for decades. Up today many important progresses have been achieved and the amount of knowledge about these topics is exponentially growing. No mention that many continuum and computational researchers have been focusing their efforts, or at least slightly getting into, in biomechanics topics during the last decade. It is relevant to point out that those theories and numerical methods used for the study of these classical materials have been used with good success when dealing with the study of biological tissue. However, biological tissues are, at some extend, much more complicated materials than those usually studied in the field of continuum mechanics, such as steel, rubbers, etc., basically due to its "living" nature. They exhibit a very optimized and advanced auto-regulatory mechanisms under mechanical and chemical stimuli that, in many cases, classical materials do not.

Bioengineers have the goal of bringing together some research fields of biology and mathematics. The basic idea is to study and understand what biologists or engineers found in its experimental studies. They have to create mathematical models that qualitatively and quantitatively describe the phenomena saw in those experiments and at the end apply to different practical goals, such as, e.g., create artificial tissues to substitute natural ones, develop clinical equipment or computational models that recreate the behavior of different tissues. The big deal of mathematical and computational models, compared with experimentation, is that once the model is correctly characterized, 
a fast and cheap reproducibility of different cases of study can be achieved as, e.g., different load cases, chemical environments, cellular disorders, etc.

The mathematical modeling of natural processes is a nice path to a better knowledge of what is happening in them. In particular, the modeling of biomechanics and mechanobiology is a enterprising area of research (??). Moreover, the inclusion of mechanical models in computational frameworks such as, e.g. the finite element method (FEM) allows researchers to study real patient specific geometries. Note that one of the main goals of computational and theoretical studies of biomechanics and mechonobiology-related problems is the application of these studies to improve current diagnostic tools and drugs, building synthetic organs (see an interesting New York Times article by ?), etc. Brought together, they are very useful tools to study many different topics within the biological and computational mechanics community.

Among many of the topics to study in biomechanics, researchers have been focusing more strongly on the modeling of different kinds of tissue as the heart (?), bones (??), vessels (????), tendoms (???), eye (??), etc. Many efforts have been also invested to model the electrical behavior of the heart (???) and arteries (?). There are also a high amount of works about the evolution of biological tissue over time, as in remodeling and growth (see e.g. the review in ? and ?). In this regard, the continuum treatment of growth (?) has been, and still is, a cornerstone in the mechanical treatment of volumetric changes in the tissue.

In the following three subsections, we briefly introduce the general mechanical approach for the constitutive modeling of biological tissues. Furthermore, we introduce the concepts of growth and remodeling from a mechanical point of view, not only looking at how the tissue behaves but also looking at how the microstructure builds-up the macroscopic behavior. We also review some computational works that deal with these approaches. We will look at the specific equations and numerical methods in the following sections. 
1.1 Mechanical behavior

A large number of constitutive laws have been proposed over the years for modeling the mechanical behavior of soft biological tissues. In general, these kind of tissues are made up of an extra-cellular matrix, composed of an isotropic ground substance with a high water content in which a network of anisotropic structures appear. The combined contribution of these constituents determines the mechanical response of the tissue, which turns out to be highly non-lineal and anisotropic. Many of numerical approaches used in the past for these phenomena, in the context of hyperelasticity, plasticity, viscoelasticity, etc., for metals or rubbers, are and have been applicable to the modeling of biological tissue (see e.g. the monographs of ????).

\subsection{Growth}

Growth has gained increasing attention in the theoretical and computational mechanics community as well (see e.g. the reviews in ????). In particular, the works of ? and ? were pioneered in the underlying kinematic description of volumetric growth. Mechanical treatment of growth has been dealt in different ways. Usually, growing and swelling of biological tissues are considered as open systems. Their different configurational settings and their numerical treatments in a finite element method (FEM) framework are discussed in ?. Typically, two fundamentally different forms of growth can be distinguished: volumetric growth and density growth, where a change of mass occurs in both of them. The first one allows for changes in volume while keeping the density constant whereas the second one maintains a constant volume while the density is allowed to change, see e.g. the works of ? in isotropic growth, ?? for anisotropic growth and ? in the context of the micro-sphere model. It is also worth noting the early work of ?, which demonstrates that the growth of blood vessels induces a change in the natural configuration of the tissue, associated with the notion of a stress free configuration. 
An alternative approach towards growth is the one presented by ?? based on mixture theory, where several constituents of a tissue are allowed to growth in an independent way. In this context other works have been presented (???) and extended to reactive mixtures (?). Although they usually stay in the theoretical description of the problem, solving the given analytical equations, ? and co-authors have step forward to implement it in a finite element framework. In a similar way, biphasic and triphasic theories (??) have been used to described different adaptation phenomena, e.g., in the brain (?) and cartilage (??) . Besides the constitutive equations and the coupling effects, there are not new numerical strategies required to solved these types of mixtures. Other interesting approaches are reported by ?? in the context of volumetric material growth.

\subsection{Remodeling}

Remodeling has been also studied from a computational and theoretical point of view. It is well known that biological tissue remodels itself when exposed to a given stimulus, e.g. mechanical loads such as an increase in blood pressure, and changes in the chemical environment that controls the signaling processes. Biological remodeling can occurs in any kind of biological tissue. In particular, the study of collagen as the most important tissue to be remodeled, in all its types (preferentially Type I and III), has been given considerable attention in the last few years (???). The reorientation of this kind of structures can be assumed to be the consequence of the reorientation of the fibrils or filaments that compose them. Several remodeling models have been proposed in the recent years. In ?, a complete consistent linearization of the equations in an implicit finite element framework was performed. Some of them analyze the reorientation of unidimensional fibers driven by different stimuli as in ? or ?.

Another important biological structure able to growth and remodel itself is the cell cytoskeleton one of the smallest and most important entities in biological matter, see e.g. ? for an overall understanding of cell behavior. Cytoskekleton is composed of microtubules, microfilaments and 
a network of actin filaments among many other elements. In terms of mechanical models there have been an increase in the number of works, see e.g. ???. Cells move and reorient their inner structure depending on the stiffness and strain of the substrate (??). These experimental results are characterized by a gradual reorientation of the principal direction of the cell followed by a progressive remodeling of the micro-structural element leading to more pointed shape (see e.g., the experimental work of ? and references therein).

In this review, we are going to go over the so called growth and remodeling of some biological tissues both from a theoretical and numerical point of view, mainly trough a continuum point of view, and within the finite element method. Growth is usually related to changes of mass (volume, density or both). We will discuss in following sections growth and remodeling of biological structures as we will provide examples of some of these approaches. We will finish discussing the most important achievements and limitations of the current approaches and we ill discuss about future lines of research.

\section{Continuum model: Balance equations and constitutive model}

During the following sections we resume the balance equation described by ? in the context of open systems. The equation sorted out hereafter are for the material parametrization and material reference, while describing the spatial motion in the context of a Lagrangian description. We refer to ?? and references therein for a complete description of balance equations in the context of hyperelastic formulation of open systems.

\subsection{Kinematics}

Following ? and ?, any quantity expressed in terms of the spatial coordinate $\mathbf{x}$ as $\{\bullet\}(\mathbf{x}, t)$ will be referred to as spatial parametrization of $\{\bullet\}$, while the material parametrization $\{\bullet\}(\mathbf{X}, t)$ is formulated in terms of the material coordinate $\mathbf{X}$. We will also distinguish between the spatial and 


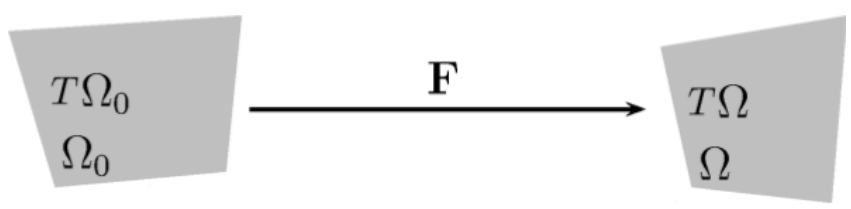

Fig. 1: Kinematic of the motion.

material reference of a scalar- or tensor-valued quantity denoted as $\{\bullet\}_{t}$, related to the spatial domain $\Omega_{t}$, or $\{\bullet\}_{0}$, related to the material domain $\Omega_{0}$. For the tensor-valued quantities, it is also possible to distinguish between the spatial, the material and the two-point description. Quantities in the spatial description are elements of the tangent or cotangent space to $\Omega_{t}$, quantities in the material description are tangent or cotangent space to $\Omega_{0}$ and tensorial quantities in the two-point description are elements of the tangent or cotangent spaces to $\Omega_{t}$ and $\Omega_{0}$. We will restrict here to the spatial motion problem, which has the physical idea of following the particles of the body from a fixed material position $\mathbf{X}$.

The spatial motion problem is characterized through the spatial motion map

$$
\mathbf{x}=\varphi(\mathbf{X}, t): \quad \Omega_{0} \rightarrow \Omega_{t}
$$

mapping the material placement $\mathbf{X}$ of a particle in $\Omega_{0}$, to the spatial placement $\mathbf{x}$ of the same particle in the spatial configuration $\Omega_{t}$, see figure ??. The related spatial deformation gradient $\mathbf{F}$ and its Jacobian $J$

$$
\mathbf{F}=\nabla_{X} \varphi(\mathbf{X}, t): \quad T \Omega_{0} \rightarrow T \Omega_{t}, \quad J=\operatorname{det} \mathbf{F}>0
$$

representing the linear tangent map from the tangent space $T \Omega_{0}$ to the time-dependent tangent space $T \Omega_{t}$. The right spatial motion Cauch- Green strain tensor $\mathbf{C}$ is defined as

$$
\mathbf{C}=\mathbf{F}^{t} \cdot \mathbf{F} \text {. }
$$


The material time derivative $\mathrm{D}_{t}$ of an arbitrary quantity $\{\bullet\}$ at fixed material placement $\mathbf{X}$ is defined as

$$
\mathrm{D}_{t}\{\bullet\}=\left.\partial_{t}\{\bullet\}\right|_{X}
$$

In this way we define the spatial velocity $\mathbf{v}$ is introduced as the material time derivative of the spatial motion map $\varphi$, as

$$
\mathbf{v}=\mathrm{D}_{t} \varphi(\mathbf{X}, t)
$$

Based on this description, we can express the material and spatial gradient as

$$
\mathrm{D}_{t} \mathbf{F}=\nabla_{X} \mathbf{v} \quad \mathbf{l}=\nabla_{x} \mathbf{v}
$$

And, e.g. the material time derivative of the spatial motion Jacobian $J$ is defined as $\mathrm{D}_{t} J=J \operatorname{div} \mathbf{v}$ with $\operatorname{div} \mathbf{v}=\mathbf{F}^{-t}: \mathrm{D}_{t} \mathbf{F}$ denoting the spatial divergence of the spatial velocity $\mathbf{v}$.

Remark 1 (Kinematics of growth) Within the previous framework, the key kinematic of finite growth can be expressed as the multiplicative decomposition of the deformation gradient $\mathbf{F}$ into an elastic part $\mathbf{F}_{e}$ and a growth part $\mathbf{F}_{g} ?$,

$$
\mathbf{F}=\mathbf{F}_{e} \cdot \mathbf{F}_{g}
$$

The underlying concept is adopted from the multiplicative decomposition in finite elastoplasticity ?. We denote the Jacobians of the elastic tensor and of the growth tensor as $J_{e}=\operatorname{det}\left(\mathbf{F}_{e}\right)$ and $J_{g}=\operatorname{det}\left(\mathbf{F}_{g}\right)$, respectively, such that $J=J_{e} J_{g}$. The elastic right Cauchy Green tensor $\mathbf{C}_{e}$ can be introduced as

$$
\mathbf{C}_{e}=\mathbf{F}_{e}^{t} \cdot \mathbf{F}_{e}=\mathbf{F}_{g}^{-t} \cdot \mathbf{C} \cdot \mathbf{F}_{g}^{-1}
$$

The pull back of the spatial velocity gradient $\mathbf{l}$ to the intermediate configuration

$$
\mathbf{F}_{e}^{-1} \cdot \mathbf{l} \cdot \mathbf{F}_{e}=\mathbf{L}_{e}+\mathbf{L}_{g}
$$

with the decomposition of the elastic contribution $\mathbf{L}_{e}$ and a growth contribution $\mathbf{L}_{g}$ defined as

$$
\mathbf{L}_{e}=\mathbf{F}_{e}^{-1} \cdot \dot{\mathbf{F}}_{e}=\dot{\mathbf{F}}_{e} \cdot \mathbf{F}_{e}^{-1} \quad \text { and } \quad \mathbf{L}_{g}=\dot{\mathbf{F}}_{g} \cdot \mathbf{F}_{g}^{-1}
$$




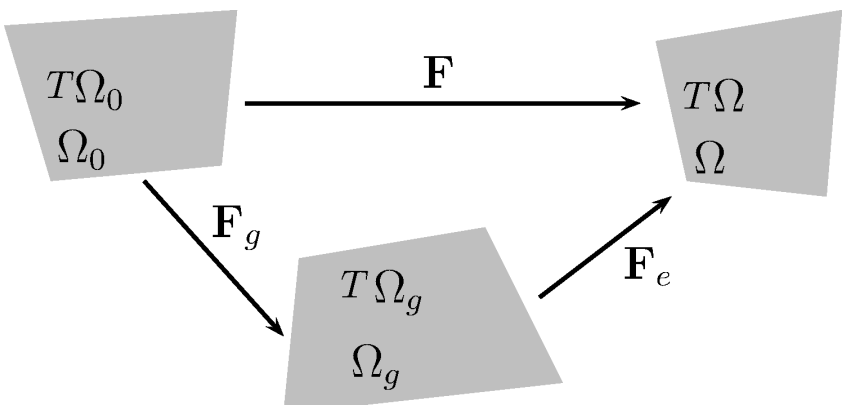

Fig. 2: Kinematic of growth. Composition of a elastic deformation gradient $\mathbf{F}_{e}$ and a growth tensor $\mathbf{F}_{g}$

Figure ?? illustrates the kinematics of finite growth, the deformation tensors $\mathbf{C}$ and $\mathbf{C}_{e}$, and the mappings $\mathbf{F}=\mathbf{F}_{e} \cdot \mathbf{F}_{g}$ between tangent spaces.

Remark 2 Growth rate

To define the evolution of the growth multiplier $\vartheta$ is usually defined, in the context of cardiovascular tissue, as the time derivative of the growth multiplier as the product of a growth-rate function and a mechanical-dependent function as

$$
\dot{\vartheta}=\kappa(\vartheta) \phi(\boldsymbol{\Xi})
$$

where $\boldsymbol{\Xi}$ represents the growth stimulus. Here, $\kappa(\vartheta)$ is a limiting function that ensures that the tissue does not grow unboundedly?,

$$
\kappa(\vartheta)=\frac{1}{\tau}\left[\frac{\vartheta^{\max }-\vartheta}{\vartheta^{\max }-1}\right]^{\gamma}
$$

with $\partial_{\vartheta} \kappa(\vartheta)=-\gamma \kappa(\vartheta) /\left[\vartheta^{\max }-\vartheta\right] . \vartheta^{\max }$ represents a growth threshold, and $\tau$ and $\gamma$ control the rate and the non-linearity of the growth process ?. The function $\phi(\boldsymbol{\Xi})$ represents the growth criterion, where the criterion variable is given by $\Xi$. 
Similarly, in the context of tumor growth, a normal assumption is to express the growth rate in terms of the Gompertz equation as

$$
\dot{\vartheta}=-\operatorname{a\vartheta log}\left(\vartheta / \vartheta_{\max }\right)
$$

\subsection{Balance of mass}

In mechanics of closed systems, the amount of mass inside a body $\Omega_{0}$ does not change. However, within the thermodynamics of open systems and in the treatment of biological tissue in particular, the mass of a body can no longer be kept constant. Therefore, the balance of mass plays a fundamental role in the modeling of biological tissue.

We express the local balance of mass as

$$
\mathrm{D}_{t} \rho_{0}=\operatorname{Div} \boldsymbol{M}+\mathcal{M}_{0}
$$

stating that the appropriate rate of change of the density $\rho_{0}$ is equal to the sum of the divergence of the related mass flux $\mathbf{M}$ and the mass source $\mathcal{M}_{0}$ (see ?? and for further details). Note that the mass fluxes $\mathbf{M}$ are actually the sum of a so-called convective contribution $\overline{\mathbf{M}}$ and the influx of mass $\mathbf{R}$ of the open system,

$$
\boldsymbol{M}=\bar{M}+\mathbf{R} \quad \text { with } \quad \bar{M}=\mathbf{0} \quad \text { for the spatial setting }
$$

The same notion apply for the extra mass sources $\mathcal{M}_{0}$ and we can expressed

$$
\mathcal{M}_{0}=\overline{\mathcal{M}}_{0}+\mathcal{R}_{0} \quad \text { with } \quad \overline{\mathcal{M}}_{0}=\mathbf{0} \quad \text { for the spatial setting }
$$

So the mass balance equation finally reads

$$
\mathrm{D}_{t} \rho_{0}=\operatorname{Div} \mathbf{R}+\mathcal{R}_{0}
$$


2.3 Balance of momentum

The mass specific version of the balance of momentum is based on the mass specific kinetic energy density, $K=\frac{1}{2} \mathbf{v} \cdot \mathbf{v}$, defining the quantity to be balanced as its partial derivative with respect to the spatial velocity $\mathbf{v}$.

$$
\mathbf{p}=\partial_{\mathbf{v}} K=\mathbf{v}
$$

The rate of change of the mass specific spatial motion momentum density $\mathbf{p}$ is balanced with the reduced momentum fluxes $\overline{\mathbf{P}}^{t}$ and the reduced momentum sources $\overline{\mathbf{b}}_{0}$ as

$$
\rho_{0} \mathrm{D}_{t} \mathbf{p}=\operatorname{Div} \overline{\mathbf{P}}^{t}+\overline{\boldsymbol{b}}_{0}
$$

where $\overline{\mathbf{P}}^{t}$ are the reduced momentum fluxes, which are related to the total momentum fluxes $\mathbf{P}^{t}$ through $\overline{\mathbf{P}}^{t}=\mathbf{P}^{t}-\mathbf{p} \otimes \mathbf{R}$. They can be expressed by the closed and open system spatial stress vector contributions $\mathbf{t}_{0}^{\text {closed }}$ and $\overline{\mathbf{t}}_{0}^{\text {open }}$ on the Neumann boundary,

$$
\overline{\mathbf{P}}^{t} \cdot \mathbf{N}=\mathbf{t}_{0}^{\text {closed }}+\overline{\mathbf{t}}_{0}^{\text {open }}
$$

Moreover, the reduced spatial momentum sources $\overline{\mathbf{b}}_{0}$ are given as the sum of the closed system volume force $\mathbf{b}_{0}^{\text {closed }}$ and the open system contributions $\overline{\mathbf{b}}_{0}^{\text {open }}$,

$$
\overline{\boldsymbol{b}}_{0}=\boldsymbol{b}_{0}^{\text {closed }}+\overline{\boldsymbol{b}}_{0}^{\text {open }}
$$

It is worth to mention that $\boldsymbol{b}_{0}^{\text {closed }}$ contributes to the external, the internal and the kinetic contributions while $\overline{\boldsymbol{b}}_{0}^{\text {open }}$ only does to the external sources. In the context of biological tissue is usual to consider the quasi-static state, so the previous equation is reduced to

$$
0=\operatorname{Div} \mathbf{P}^{t}+\overline{\boldsymbol{b}}_{0}
$$


2.4 Balance of kinetic energy

To investigate the mass specific version of the balance of kinetic energy, we need to evaluate the material time derivative of the mass specific kinetic energy density $K=1 / 2 \mathbf{v} \cdot \mathbf{g} \cdot \mathbf{v}$ as

$$
\rho_{0} \mathrm{D}_{t} K=\mathrm{D}_{t} K_{0}-K \mathrm{D}_{t} \rho_{0}
$$

and identifying the mass specific external and internal mechanical power $\overline{\mathrm{p}}_{0}^{e x t}$ and $\overline{\mathrm{p}}_{0}^{i n t}$, and keeping in mind that the internal force term $\overline{\boldsymbol{b}}_{0}^{\text {int }}=\mathbf{0}$ and the convective mass vanishes identically for the spatial motion case, as

$$
\begin{aligned}
& \overline{\mathrm{p}}_{0}^{e x t}:=\mathrm{p}_{0}^{e x t}-K \mathrm{D}_{t} \rho_{0}=\operatorname{Div}\left(\mathbf{v} \cdot \overline{\mathbf{P}}^{t}\right)+\mathbf{v} \cdot \overline{\boldsymbol{b}}_{0}^{\text {ext }} \\
& \overline{\mathrm{p}}_{0}^{i n t}:=\mathrm{p}_{0}^{i n t} \quad=\overline{\boldsymbol{P}}^{t}: \mathrm{D}_{t} \mathbf{F}
\end{aligned}
$$

With the above definitions at hand, the mass specific balance of kinetic energy can be expressed in the following form.

$$
\rho_{0} \mathrm{D}_{t} K=\overline{\mathrm{p}}_{0}^{e x t}-\overline{\mathrm{p}}_{0}^{\text {int }}
$$

\subsection{Balance of energy}

In contrast with closed systems, where the external power is only due to a purely mechanical contribution $\mathrm{p}_{0}^{\text {ext }}$ and a thermal contribution $\mathrm{q}_{0}^{e x t}$, in open systems an additional external open system contribution have to be added in the definition of the non-mechanical external power $\mathrm{q}_{0}^{e x t}$.

The mass specific energy density $E$ balances with the external mechanical power $\bar{p}_{0}^{e x t}$ and the non-mechanical power $\overline{\mathrm{q}}_{0}^{\text {ext }}$ as

$$
\rho_{0} \mathrm{D}_{t} E=\overline{\mathrm{p}}_{0}^{e x t}+\overline{\mathrm{q}}_{0}^{e x t}
$$

The corresponding balance equation reads

$$
\rho_{0} \mathrm{D}_{t} I=\overline{\mathrm{p}}_{0}^{i n t}+\overline{\mathrm{q}}_{0}^{e x t}
$$


where

$$
\overline{\mathrm{q}}_{0}^{e x t}:=\mathrm{q}_{0}^{e x t}-I \mathrm{D}_{t} \rho_{0}+=-\operatorname{Div} \overline{\boldsymbol{Q}}+\overline{\mathcal{Q}}_{0} .
$$

Again, we can relate the reduced energy fluxes $\overline{\mathbf{Q}}$ defined through $\overline{\mathbf{Q}}=\mathbf{Q}-I \mathbf{R}$ to the classical heat flux $q_{0}^{\text {closed }}$ and the energy flux caused by additional effects of the open system $\bar{q}_{0}^{\text {open }}$.

$$
\overline{\mathbf{Q}} \cdot \mathbf{N}=q_{0}^{\text {closed }}+\bar{q}_{0}^{\text {open }}
$$

In the same way, the reduced non-mechanical energy sources $\overline{\mathcal{Q}}_{0}$ are given as the sum of the classical heat source of a closed system $\mathcal{Q}_{0}^{\text {closed }}$ and the additional open system contribution to the energy $\overline{\mathcal{Q}}_{0}^{\text {open }}$.

$$
\overline{\mathcal{Q}}_{0}=\mathcal{Q}_{0}^{\text {closed }}+\overline{\mathcal{Q}}_{0}^{\text {open }}
$$

2.6 Balance of entropy and dissipation inequality

Also known as the second law of thermodynamics, the balance of entropy establish the internal production of a specific entropy required to either vanish for reversible processes or to be strictly positive for irreversible processes.

The mass specific version establish that the rate of change of the entropy $S$ have to be in equilibrium with the external entropy income $\overline{\mathrm{h}}_{0}^{\text {ext }}$ and the internal entropy increase $\overline{\mathrm{h}}_{0}^{\text {int }}$

$$
\rho_{0} \mathrm{D}_{t} S=\overline{\mathrm{h}}_{0}^{e x t}+\overline{\mathrm{h}}_{0}^{i n t}
$$

such that

$$
\begin{aligned}
& \overline{\mathrm{h}}_{0}^{\text {ext }}:=\mathrm{h}_{0}^{\text {ext }}-S \mathrm{D}_{t} \rho_{0}=-\operatorname{Div} \overline{\boldsymbol{H}}+\overline{\mathcal{H}}_{0} \\
& \overline{\mathrm{h}}_{0}^{\text {int }}:=\mathrm{h}_{0}^{\text {int }} \geq 0
\end{aligned}
$$

where the reduced entropy fluxes $\overline{\mathbf{H}}=\mathbf{H}-S \mathbf{R}$ can be related to the entropy flux of closed systems, $h_{0}^{\text {closed }}$, and the additional effects of the open system, $\bar{h}_{0}^{\text {open }}$, as

$$
\overline{\boldsymbol{H}} \cdot \mathbf{N}=h_{0}^{\text {closed }}+\bar{h}_{0}^{\text {open }}
$$


In the same way, the reduced entropy sources $\overline{\mathcal{H}}_{0}=\mathcal{H}_{0}-S \mathcal{R}_{0}+\nabla_{X} S \cdot \mathbf{R}$ can be related to closed and open, $\mathcal{H}_{0}^{\text {closed }}$ and $\overline{\mathcal{H}}_{0}^{\text {open }}$ respectively, as

$$
\overline{\mathcal{H}}_{0}=\mathcal{H}_{0}^{\text {closed }}+\overline{\mathcal{H}}_{0}^{\text {open }}
$$

Reformulating the above second law form, we obtain the dissipation inequality that state restrictions on the constitutive functions. The dissipation rate can be expresses as the internal entropy production weighted by the absolute temperature as $\overline{\mathrm{d}}_{0}:=\theta \overline{\mathrm{h}}_{0}^{\text {int }} \geq 0$. The dissipation rate in the spatial motion version of the Clausius- Duhem inequality, given a isothermal process, can be given as

$$
\overline{\mathrm{d}}_{0}=\overline{\mathrm{p}}_{0}^{i n t}-\rho_{0} \mathrm{D}_{t}[I-\theta S]-\mathrm{s}_{0} \theta \geq 0
$$

And finally, using the Legendre transform, the Helmholtz free energy $\Psi=I-\theta S$ can be obtained, ending up with the free energy-function of the Clausius- Duhem inequality.

$$
\overline{\mathrm{d}}_{0}=\overline{\boldsymbol{P}}^{t}: \mathrm{D}_{t} \mathbf{F}-\mathbf{v} \cdot \overline{\mathbf{b}}_{0}^{\text {int }}-\rho_{0} \mathrm{D}_{t} \Psi-\mathrm{s}_{0} \theta \geq 0
$$

Note that, considering the usual quasi-static nature of biological tissues we can consider $\mathbf{v} \cdot \overline{\mathbf{b}}_{0}^{\text {int }}=0$.

\subsection{Constitutive model}

The thermodynamic treatment of any type of biological tissues is a complex task. In the context of hyperelasticity, which is the general framework in the modeling of biological tissues, the definition of a given material relies on the definition of a strain energy density function (SEDF) $\Psi$. In this section we review the usual definition of a thermodynamically consistent approach to characterize the material response (??). It is common in the context of constitutive modeling of biological tissue to formulate the problem in terms of material version of the Clasius-Planck inequality for isothermal processes, which is a reasonable assumption for biological tissue with a relatively constant temperature, as 


$$
\overline{\mathrm{D}}_{0}=\frac{1}{2} \mathbf{S}: \dot{\mathbf{C}}-\rho_{0} \mathrm{D}_{t} \Psi-\mathrm{S}_{0} \theta \geq 0
$$

where $\mathrm{S}_{0}$ is the material form of internal dissipation and entrophy respectively. $\mathbf{S}$ represents the second Piola-Kirchoff stress tensor and $\mathbf{C}$ is the right Cauchy-Green tensor.

Usually in the modeling of biological soft tissue the SEDF is split as $\Psi=\Psi_{\mathrm{vol}}+\Psi_{\mathrm{ich}}=\Psi_{\mathrm{vol}}+$ $\Psi_{\text {iso }}+\Psi_{\text {ani }}$. The first term, $\Psi_{\text {vol }}$, characterizes the volumetric part of the energy and can be used to impose quasi-incompressibility of soft tissues due to its high water content. The remaining term, $\Psi_{\mathrm{ich}}$, characterizes the deviatoric or isochoric part of the SEDF. The isochoric part can be further decomposed into an isotropic part, $\Psi_{\text {iso }}$ and a anisotropic term $\Psi_{\text {ani }}$.

From its material derivative

$$
\dot{\Psi}=\left[\partial_{\mathbf{C}} \Psi_{\mathrm{vol}}+\partial_{\mathbf{C}} \Psi_{\text {iso }}+\partial_{\mathbf{C}} \Psi_{\mathrm{ani}}\right]: \dot{\mathbf{C}}+\partial_{\rho} \Psi \dot{\rho}
$$

we can obtain the expression for the Piola-Kirchhoff stress tensor (?) from Eq. ?? as

$$
\mathbf{S}=2 \partial_{\mathbf{C}} \Psi_{\mathrm{vol}}+2 \partial_{\mathbf{C}} \Psi_{\mathrm{iso}}+2 \partial_{\mathbf{C}} \Psi_{\mathrm{ani}}=\mathbf{S}_{\mathrm{vol}}+\mathbf{S}_{\mathrm{iso}}+\mathbf{S}_{\mathrm{ani}}
$$

or, more specifically, for the quasi-incompressibility assumption and in a invariant-based approach

$$
\mathbf{S}=2 \partial_{\partial J} \Psi_{\operatorname{vol}(J)} \partial J_{\mathbf{C}}+2\left[\partial_{\bar{I}_{1}} \Psi \operatorname{iso} \partial_{\overline{\mathbf{C}}} \bar{I}_{1}+\partial_{\bar{I}_{4}} \Psi_{\text {iso }} \partial_{\overline{\mathbf{C}}} \bar{I}_{4}\right]: \partial_{\mathbf{C}} \overline{\mathbf{C}}
$$

Here, it is common to introduce the deviatoric projection tensor $\mathbb{P}=\mathbb{I}-\frac{1}{3} \mathbf{C}^{-1} \otimes \mathbf{C}$, such that $\partial_{\mathbf{C}} \overline{\mathbf{C}}=J^{-2 / 3} \mathbb{P}^{t}$, where $\mathbb{I}=\delta_{i k} \delta_{j l} \mathbf{e}_{i} \otimes \mathbf{e}_{j} \otimes \mathbf{e}_{k} \otimes \mathbf{e}_{l}$ represents the fourth order identity tensor. Accordingly, the purely deviatoric part of the second Piola-Kirchoff stress tensor, $\mathbf{S}_{\mathrm{ich}}$, can be expressed as

$$
\mathbf{S}_{\mathrm{ich}}=J^{-2 / 3} \mathbb{P}: \overline{\mathbf{S}}_{\mathrm{ich}}=J^{-2 / 3} \operatorname{DEV}\left(\overline{\mathbf{S}}_{\mathrm{ich}}\right),
$$

where $\overline{\mathbf{S}}_{\mathrm{ich}}=2 \partial_{\overline{\mathbf{C}}} \Psi_{\mathrm{ich}}$, and $\operatorname{DEV}(\bullet)=\left[\mathbb{I}-\frac{1}{3} \mathbf{C}^{-1} \otimes \mathbf{C}\right]:(\bullet)=\mathbb{P}:(\bullet)$ is the deviatoric operator in the material description.

Also, the tensor of tangent moduli that relates stress and strain increments, is essential for a consistent finite element implementation. It is given by 


$$
\mathbf{C}=2 \mathrm{~d}_{\mathbf{C}} \mathbf{S}=\mathbf{C}_{\mathrm{vol}}+\mathbf{C}_{\mathrm{ich}}
$$

Where the volumetric and isochoric contributions read

$$
\begin{aligned}
\mathbf{C}_{\mathrm{vol}}=2 \mathrm{~d}_{\mathbf{C}} \mathbf{S}_{\mathrm{vol}} & =2 \mathrm{~J} \tilde{p} \mathbf{C}^{-1} \otimes \mathbf{C}^{-1}-2 \mathrm{~J} p \mathbb{I}_{\mathrm{C}^{-1}}, \\
\mathbf{C}_{\mathrm{ich}}=2 \mathrm{~d}_{\mathbf{C}} \mathbf{S}_{\mathrm{ich}} & =\mathbb{P}: \overline{\mathbf{C}}: \mathbb{P}^{\mathrm{t}}-\frac{2}{3} \operatorname{tr}\left(\mathrm{J}^{-2 / 3} \mathbf{S}_{\mathrm{iso}}\right) \tilde{\mathbb{P}} \\
& -\frac{2}{3}\left[\overline{\mathbf{S}} \otimes \mathbf{C}^{-1}+\mathbf{C}^{-1} \otimes \overline{\mathbf{S}}\right],
\end{aligned}
$$

Here, $p$ denotes the hydrostatic pressure and $\tilde{p}=p+\mathrm{J} \partial_{\mathrm{J}} p$, with the fourth order tensors $\tilde{\mathbb{P}}=\mathbb{I}_{\mathrm{C}^{-1}}-\frac{1}{3} \mathbf{C}^{-1} \otimes \mathbf{C}^{-1}$ and $\mathbb{I}_{\mathrm{C}^{-1}}=\frac{1}{2}\left[\mathbf{C}^{-1} \bar{\otimes} \mathbf{C}^{-1}+\mathbf{C}^{-1} \underline{\otimes} \mathbf{C}^{-1}\right]$, where the non-standard fourth order tensor products can be expressed as $\{\bullet \bar{\otimes} \circ\}_{i j k l}=\{\bullet\}_{i k}\{\circ\}_{j l}$ and $\left\{\bullet \underline{\otimes}^{\circ}\right\}_{i j k l}=\{\bullet\}_{i l}\{\circ\}_{j k}$.

Remark 3 (Constitutive equations of growth) To take into account the kinematics of growth into the hyperelastic description introduced here. We write the Helmholtz strain energy function $\Psi$, which was initially parameterized in terms of the elastic deformation tensor $\mathbf{C}_{\mathrm{e}}$, in terms of the total deformation tensor $\mathbf{C}$ and the growth tensor $\mathbf{F}_{\mathrm{g}}$, such that

$$
\dot{\Psi}=\partial_{\mathbf{C}} \Psi: \dot{\mathbf{C}}+\partial_{\mathbf{F g}_{\mathrm{g}}} \Psi: \dot{\mathbf{F}}_{\mathrm{g}}
$$

The tangent moduli is given as follows.

$$
\begin{aligned}
\mathbf{C}=2 d_{\mathbf{C}} \mathbf{S} & =\mathbf{C}_{\mathrm{e}}+\mathbf{C}_{\mathrm{g}} \\
& =\left.2 \partial_{\mathbf{C}} \mathbf{S}\right|_{\mathbf{F}_{\mathrm{g}}}+\left.2 \partial_{\mathbf{C}} \mathbf{S}\right|_{\mathbf{F}} \\
& =\left.2 \partial_{\mathbf{C}} \mathbf{S}\right|_{\mathbf{F}_{\mathrm{g}}}+\left.2\left[\partial_{\mathbf{F g}} \mathbf{S}: \partial_{\vartheta} \mathbf{F}_{\mathrm{g}}\right] \otimes \partial_{\mathbf{C}} \vartheta\right|_{\mathbf{F}}
\end{aligned}
$$

The first term of equation (??) represents the previously described classical elastic part, $2 d_{\mathbf{C}_{\mathrm{e}}} \mathbf{S}_{\mathrm{e}}$, pulled back to the undeformed reference configuration,

$$
\mathbf{C}_{\mathrm{e}}=\left.2 d_{\mathbf{C}} \mathbf{S}\right|_{\mathbf{F}_{\mathrm{g}}}=\left[\mathbf{F}_{\mathrm{g}}^{-1} \bar{\otimes} \mathbf{F}_{\mathrm{g}}^{-1}\right]: 2 d_{\mathbf{C}_{\mathrm{e}}} \mathbf{S}_{\mathrm{e}}:\left[\mathbf{F}_{\mathrm{g}}^{-\mathrm{t}} \bar{\otimes} \mathbf{F}_{\mathrm{g}}^{-\mathrm{t}}\right]
$$


The second term of equation (??) is due to the grow part of the formulation.

$$
\mathbf{C}_{\mathrm{g}}=\left.2 d_{\mathbf{C}} \mathbf{S}\right|_{\mathbf{F}}=\left.2\left[\partial_{\mathbf{F}_{\mathrm{g}}} \mathbf{S}: \partial_{\vartheta} \mathbf{F}_{\mathrm{g}}\right] \otimes \partial_{\mathbf{C}} \vartheta\right|_{\mathbf{F}}
$$

where

$$
\begin{aligned}
\partial_{\mathbf{F}_{\mathrm{g}}} \mathbf{S}= & -\left[\mathbf{F}_{\mathrm{g}}^{-1} \bar{\otimes} \mathbf{S}+\mathbf{S} \underline{\otimes} \mathbf{F}_{\mathrm{g}}^{-1}\right] \\
& -\left[\mathbf{F}_{\mathrm{g}}^{-1} \bar{\otimes} \mathbf{F}_{\mathrm{g}}^{-1}\right]: \frac{1}{2} \mathbf{C}:\left[\mathbf{F}_{\mathrm{g}}^{-\mathrm{t}} \bar{\otimes} \mathbf{C}_{\mathrm{e}}+\mathbf{C}_{\mathrm{e}} \otimes \mathbf{F}_{\mathrm{g}}^{-\mathrm{t}}\right],
\end{aligned}
$$

The terms $\partial_{\vartheta} \mathbf{F}_{\mathrm{g}}$ and $\partial_{\mathbf{F}} \vartheta$ are specific of the chosen growth tensor $\mathbf{F}_{\mathrm{g}}$.

\section{Finite element formulation}

In the following, we shall illustrate the derivation of a finite element formulation for open systems restricting ourselves to the isothermal case. The finite element method is by far the most widely method used to deal, numerically, with the simulation of the continuum biological models described in the previous section. The reader is addressed to ?, where a more complete version of the following section can be found. The density $\rho_{0}$ and the deformation $\varphi$ state the primary unknowns of the resulting two-field formulation which will be solved in a monolithic sense.

3.1 Strong form of the coupled boundary value problem

The reference density $\rho_{0}$ and the deformation map $\varphi$ primary variable of the problem and are governed by the balance of mass (??) and the mass specific balance of linear momentum (??). The residual forms $r^{\rho}=0$ and $r^{\varphi}=\mathbf{0}$ for the material domain $\Omega_{0}$ can be given as

$$
\begin{aligned}
& \mathrm{r}^{\rho}=\mathrm{D}_{t} \rho_{0}-\operatorname{Div} \boldsymbol{R}-\mathcal{R}_{0}=0 \quad \text { in } \Omega_{0} \\
& r^{\varphi}=\rho_{0} D_{t} \boldsymbol{p}-\operatorname{Div} \overline{\mathbf{P}}^{t}-\overline{\boldsymbol{b}}_{0}=\mathbf{0} \quad \text { in } \Omega_{0}
\end{aligned}
$$

The boundary $\partial \Omega_{0}$ of the material domain is split into disjoint parts $\partial \Omega_{0}^{\rho}$ and $\partial \Omega_{0}^{r}$ for the density and the for the deformation problem, $\partial \Omega_{0}^{\varphi}$ and $\partial \Omega_{0}^{t}$.

$$
\begin{array}{ll}
\partial \Omega_{0}^{\rho} \cup \partial \Omega_{0}^{r}=\partial \Omega_{0} & \partial \Omega_{0}^{\rho} \cap \partial \Omega_{0}^{r}=\emptyset \\
\partial \Omega_{0}^{\varphi} \cup \partial \Omega_{0}^{t}=\partial \Omega_{0} & \partial \Omega_{0}^{\varphi} \cap \partial \Omega_{0}^{t}=\emptyset
\end{array}
$$


Dirichlet boundary conditions are prescribed on $\partial \Omega_{0}^{\rho}$ and $\partial \Omega_{0}^{\varphi}$, while Neumann boundary conditions are on $\partial \Omega_{0}^{r}$ and $\partial \Omega_{0}^{t}$.

$$
\begin{aligned}
& \rho_{0}=\bar{\rho}_{0} \quad \text { on } \quad \partial \Omega_{0}^{\rho} \quad \boldsymbol{R}\left(\rho_{0}, \boldsymbol{\varphi}\right) \cdot \boldsymbol{N}=r^{\text {closed }}+\bar{r}^{\text {open }} \quad \text { on } \quad \partial \Omega_{0}^{r} \\
& \boldsymbol{\varphi}=\overline{\boldsymbol{\varphi}} \quad \text { on } \quad \partial \Omega_{0}^{\varphi} \quad \overline{\mathbf{P}}^{t}\left(\rho_{0}, \boldsymbol{\varphi}\right) \cdot \boldsymbol{N}=\boldsymbol{t}^{\text {closed }}+\overline{\boldsymbol{t}}^{\text {open }} \quad \text { on } \quad \partial \Omega_{0}^{r}
\end{aligned}
$$

3.2 Weak form of the coupled boundary value problem

The reformulation of the strong form into the weak form, the balance of mass (??) 1 and the corresponding Neumann boundary conditions $(? ?)_{2}$ are tested by the scalar-valued test function $\delta \rho$. For the residual of the balance of linear momentum $(? ?)_{2}$ and the related Neumann boundary conditions (??) 4 , the vector-valued test function $\delta \varphi$ are used to obtain

$$
\begin{aligned}
& \mathrm{R}^{\rho}=\int_{\Omega_{0}}^{\delta \rho} \quad \mathrm{D}_{t} \rho_{0} \mathrm{~d} V+\int_{\Omega_{0}} \nabla_{X} \delta \rho \boldsymbol{R} \mathrm{d} V-\int_{\partial \Omega_{0}^{r}} \delta \rho \quad\left[r^{\text {closed }}+\bar{r}^{\text {open }}\right] \mathrm{d} A-\int_{\Omega_{0}} \delta \rho \mathcal{R}_{0}=0 \forall \delta \rho \text { in } H_{1}^{0}\left(\Omega_{0} \mathrm{~d} V\right. \\
& \mathrm{R}^{\varphi}=\int_{\Omega_{0}} \delta \boldsymbol{\varphi} \cdot \rho_{0} \mathrm{D}_{t} \boldsymbol{p} \mathrm{d} V+\int_{\Omega_{0}} \nabla_{X} \delta \boldsymbol{\varphi} \overline{\mathbf{P}}^{t} \mathrm{~d} V-\int_{\partial \Omega_{0}^{t}} \delta \boldsymbol{\varphi} \cdot\left[\boldsymbol{t}^{\text {closed }}+\overline{\boldsymbol{t}}^{\text {open }}\right] \mathrm{d} A-\int_{\Omega_{0}} \delta \boldsymbol{\varphi} \cdot \overline{\boldsymbol{b}}_{0}=0 \forall \delta \boldsymbol{\varphi} \text { in } H_{1}^{0}\left(\Omega_{0} \mathrm{~d} V\right.
\end{aligned}
$$

3.3 Temporal discretization of the coupled boundary value problem

For the temporal discretization of the previous equations, the usual time interval of interest $T=$ $\bigcup_{n=0}^{n_{\text {step }}-1}\left[t_{n}, t_{n+1}\right]$ is considered. The time subinterval $\left[t_{n}, t_{n+1}\right]$ where $\Delta t:=t_{n+1}-t_{n}>0$ is the actual time increment. Given the primary unknowns, $\rho_{0} n$ and $\varphi_{n}$, and the derivable quantities at $t_{n}$, a classical implicit Euler backward time integration scheme is used to establish the equations in terms of the unknowns $\rho_{0 n+1}$ and $\boldsymbol{\varphi}_{n+1}$. Given the following approximation of the first order material time derivatives

$$
\mathrm{D}_{t} \rho_{0}=\frac{1}{\Delta t}\left[\rho_{0} n+1-\rho_{0 n}\right] \quad \mathrm{D}_{t} \boldsymbol{p}=\frac{1}{\Delta t}\left[\boldsymbol{p}_{n+1}-\boldsymbol{p}_{n}\right]
$$


the residuals read

$$
\begin{aligned}
& \mathrm{R}_{n+1}^{\rho}\left(\delta \rho ; \rho_{0} n+1, \varphi_{n+1}\right)=0 \quad \forall \quad \delta \rho \text { in } H_{1}^{0}\left(\Omega_{0}\right) \\
& \mathrm{R}_{n+1}^{\varphi}\left(\delta \boldsymbol{\varphi} ; \rho_{0 n+1}, \varphi_{n+1}\right)=0 \quad \forall \quad \delta \varphi \text { in } H_{1}^{0}\left(\Omega_{0}\right)
\end{aligned}
$$

we obtain the semi-discrete virtual work expressions of the balance of mass and linear momentum as

$$
\begin{array}{rlrl}
\mathrm{R}_{n+1}^{\rho} & =\int_{\Omega_{0}} \delta \rho & \frac{\rho_{0 n+1}-\rho_{0 n}}{\Delta t}+ & \nabla_{X} \delta \rho \cdot \boldsymbol{R}_{n+1} \mathrm{~d} V \\
& -\int_{\partial \Omega_{0}^{r}}^{\delta \rho} & {\left[r_{n+1}^{\text {closed }}+\bar{r}_{n+1}^{\text {open }}\right] \mathrm{d} A} & -\int_{\Omega_{0}} \delta \rho \mathcal{R}_{0 n+1} \mathrm{~d} V=0 \forall \delta \rho \operatorname{in} H_{1}^{0}\left(\Omega_{0}\right) \\
\mathrm{R}_{n+1}^{\varphi} & =\int_{\Omega_{0}} \delta \boldsymbol{\varphi} \cdot \rho_{0} \frac{\boldsymbol{p}_{n+1}-\boldsymbol{p}_{n}}{\Delta t}+\quad \nabla_{X} \delta \boldsymbol{\varphi}: \overline{\mathbf{P}}_{n+1}^{t} \mathrm{~d} V \\
& -\int_{\partial \Omega_{0}^{t}} \delta \boldsymbol{\varphi} & \cdot\left[\boldsymbol{t}_{n+1}^{\text {closed }}+\overline{\boldsymbol{t}}_{n+1}^{\text {open }}\right] \mathrm{d} A & -\int_{\Omega_{0}} \delta \boldsymbol{\varphi} \cdot \overline{\boldsymbol{b}}_{0 n+1} \mathrm{~d} V=0 \forall \delta \boldsymbol{\varphi i n} H_{1}^{0}\left(\Omega_{0}\right)
\end{array}
$$

3.4 Spatial discretization of the coupled boundary value problem

In terms of the spatial discretization with the finite element method the underlying domain $\Omega_{0}$ is discretized into $n_{e l}$ elements $\Omega_{0}^{e}$, whereby the underlying geometry is interpolated by the shape functions $N_{X}^{i}$ in terms of the nodal point positions $\boldsymbol{X}_{i}$ of the $i=1 . . n_{e l}$ with $n_{e l}$ the element nodes.

$$
\Omega_{0}=\left.\bigcup_{e=1}^{n_{e l}} \Omega_{0}^{e} \quad \boldsymbol{X}^{h}\right|_{\Omega_{0}^{e}}=\sum_{I=1}^{n_{e l}} N_{X}^{i} \boldsymbol{X}_{i}
$$

The full set of $n_{n p}$ global node points $\mathbb{B}$ can be gathered to the complete set of $n_{e l} \mathbb{B}^{e}$ as $\mathbb{B}=\bigcup_{e=1}^{n_{e l}} \mathbb{B}^{e}$ with $\mathbb{B}=\left\{I \mid I=1, n_{n p}\right\}$ and $\mathbb{B}^{e}=\left\{i \mid i=1, n_{e l}\right\}$ citepKuhl2003b.

The unknowns variables $\rho_{0}$ and $\varphi$ can be interpolated on the element with the same shape functions $N_{\rho}^{i}$ and $N_{\varphi}^{j}$ following an isoparametric approach. And they are also used to interpolate the test functions $\delta \rho$ and $\delta \varphi$ as

$$
\begin{array}{rlll}
\left.\delta \rho^{h}\right|_{\Omega_{0}^{e}}=\sum_{i=1}^{n_{e l}} N_{\rho}^{i} \delta \rho_{i} & \in H_{1}^{0}\left(\Omega_{0}\right) & \left.\rho_{0}^{h}\right|_{\Omega_{0}^{e}}=\sum_{k=1}^{n_{e l}} N_{\rho}^{k} & \rho_{k}=0 \in H_{1}\left(\Omega_{0}\right) \\
\left.\delta \varphi^{h}\right|_{\Omega_{0}^{e}}=\sum_{j=1}^{n_{e l}} N_{\varphi}^{j} \delta \varphi_{j} & \in H_{1}^{0}\left(\Omega_{0}\right) & \left.\varphi^{h}\right|_{\Omega_{0}^{e}}=\sum_{l=1}^{n_{e l}} N_{\varphi}^{l} & \varphi_{l}=0 \in H_{1}\left(\Omega_{0}\right)
\end{array}
$$


In the same fashion the gradients of the test functions $\nabla_{X} \delta \rho^{h}$ and $\nabla_{X} \delta \varphi^{h}$ and the gradients of $\nabla_{X} \rho^{h}$ and $\nabla_{X} \varphi^{h}$ takes the following format.

$$
\begin{array}{ll}
\left.\nabla_{X} \delta \rho^{h}\right|_{\Omega_{0}^{e}}=\sum_{i=1}^{n_{e l}} \delta \rho_{i} \quad \nabla_{X} N_{\rho}^{i} & \left.\nabla_{X} \rho_{0}^{h}\right|_{\Omega_{0}^{e}}=\sum_{k=1}^{n_{e l}} \rho_{k} \nabla_{X} N_{\rho}^{k} \\
\left.\nabla_{X} \delta \varphi^{h}\right|_{\Omega_{0}^{e}}=\sum_{j=1}^{n_{e l}} \delta \varphi_{j} \otimes \nabla_{X} N_{\varphi}^{j} & \left.\nabla_{X} \varphi^{h}\right|_{\Omega_{0}^{e}}=\sum_{l=1}^{n_{e l}} \varphi_{l} \otimes \nabla_{X} N_{\varphi}^{l}
\end{array}
$$

Based on the previous discretization of the primary unknowns, the discrete balance of mass and momentum are defined as

$$
\begin{array}{lll}
\mathrm{R}_{I}^{\rho}\left(\rho_{0 n+1}^{h}, \varphi_{n+1}^{h}\right) & =0 & \forall I \text { in } \mathbb{B} \\
\mathbf{R}_{J}^{\varphi}\left(\rho_{0 n+1}^{h}, \varphi_{n+1}^{h}\right)=\mathbf{0} & \forall J \text { in } \mathbb{B}
\end{array}
$$

whereby the discrete scalar-valued residuum of the balance of mass $\mathrm{R}_{I}^{\rho}$ and the discrete vectorvalued residuum of the balance of momentum $\mathbf{R}_{J}^{\varphi}$ expand to the following expressions.

$$
\begin{aligned}
& \mathrm{R}_{I}^{\rho}={\underset{e=1}{n_{e l}}}_{\Omega_{0}^{e}} N_{\rho}^{i} \quad \frac{\rho_{0 n+1}-\rho_{0 n}}{\Delta t}+\nabla_{X} N_{\rho}^{i} \cdot \quad \boldsymbol{R}_{n+1} \mathrm{~d} V \\
& -\int_{\partial \Omega_{0}^{e r}} N_{\rho}^{i}\left[r_{n+1}^{\text {closed }}+\bar{r}_{n+1}^{\text {open }}\right] \quad \mathrm{d} A-\int_{\Omega_{0}^{e}} N_{\rho}^{i} \mathcal{R}_{0 n+1} \mathrm{~d} V=\forall I \quad \text { in } \quad \mathbb{B} \\
& \mathbf{R}_{J}^{\varphi}={\stackrel{\text { A }}{n_{e l}}}_{e=1}^{\int_{\Omega_{0}^{e}} N_{\varphi}^{j}} \quad \rho_{0} \frac{\boldsymbol{p}_{n+1}-\boldsymbol{p}_{n}}{\Delta t}+\nabla_{X} N_{\varphi}^{j} \cdot \quad \overline{\mathbf{P}}_{n+1} \mathrm{~d} V \\
& -\int_{\partial \Omega_{0}^{t e}} N_{\varphi}^{j}\left[\boldsymbol{t}_{n+1}^{\text {closed }}+\overline{\boldsymbol{t}}_{n+1}^{\text {open }}\right] \quad \mathrm{d} A-\int_{\Omega_{0}^{e}} N_{\varphi}^{j} \overline{\boldsymbol{b}}_{0 n+1} \mathrm{~d} V=\boldsymbol{\bullet} J \quad \text { in } \quad \mathbb{B}
\end{aligned}
$$

As described in ? the operator $\mathbf{A}$ denotes the assembly of all element contributions at the element nodes $i, j=1, n_{e l}$ to the overall residuals at the global node points $I, J=1, n_{n p}$.

\subsection{Linearization of the coupled boundary value problem}

To solve the above nonlinear coupled system of equations (??), a monolithic incremental iterative Newton Raphson technique is usually used. The consistent linearization of those equations at time 
$t_{n+1}$ and the iterative residual, $\mathrm{dR}_{I}^{\rho}$ and $\mathrm{d} \mathbf{R}_{J}^{\varphi}$, are presented.

$$
\begin{aligned}
& \mathrm{R}_{I n+1}^{\rho k+1}=\mathrm{R}_{I n+1}^{\rho k}+\mathrm{dR}_{I}^{\rho} \doteq 0 \quad \forall \quad I \quad \text { in } \quad \mathbb{B} \\
& \mathbf{R}_{J n+1}^{\varphi k+1}=\mathbf{R}_{J n+1}^{\varphi k}+\mathrm{d} \mathbf{R}_{J}^{\varphi} \doteq \mathbf{0} \quad \forall \quad J \quad \text { in } \quad \mathbb{B} \\
& \mathrm{dR}_{I}^{\rho}=\sum_{K=1}^{n_{n p}} \mathrm{~K}_{\rho \rho}^{I K} \mathrm{~d} \rho_{K}+\sum_{L=1}^{n_{n p}} \mathbf{K}_{\rho \varphi}^{I L} \cdot \mathrm{d} \boldsymbol{\varphi}_{L} \quad \forall \quad I \quad \text { in } \quad \mathbb{B} \\
& \mathrm{d} \mathbf{R}_{J}^{\varphi}=\sum_{K=1}^{n_{n p}} \mathbf{K}_{\varphi \rho}^{J K} \mathrm{~d} \rho_{K}+\sum_{L=1}^{n_{n p}} \mathbf{K}_{\varphi \varphi}^{J L} \cdot \mathrm{d} \boldsymbol{\varphi}_{L} \quad \forall \quad J \quad \text { in } \mathbb{B}
\end{aligned}
$$

The above global tangent matrices, $\mathrm{K}_{\rho \rho}^{I K}, \mathbf{K}_{\rho \varphi}^{I L}, \mathbf{K}_{\varphi \rho}^{J K}$ and $\mathbf{K}_{\varphi \varphi}^{J L}$, can be expressed as

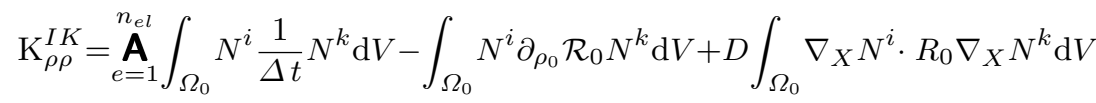

$$
\begin{aligned}
& \mathbf{K}_{\rho \varphi}^{I L}=\stackrel{n_{e=1}}{\mathbf{A}}-\int_{\Omega_{0}} N^{i} \partial_{\boldsymbol{F}} \mathcal{R}_{0} \cdot \nabla_{X} N^{l} \mathrm{~d} V
\end{aligned}
$$

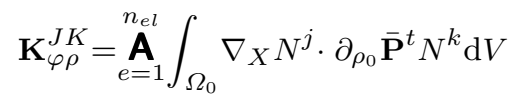

$$
\begin{aligned}
& \mathbf{K}_{\varphi \varphi}^{J L}=\stackrel{n_{e l}}{\stackrel{A}{A}} \int_{\Omega_{0}} N^{j} \rho_{0} \frac{1}{\Delta t} \boldsymbol{I} N^{l} \mathrm{~d} V+\int_{\Omega_{t}} \nabla_{X} N^{j} \cdot \partial_{\boldsymbol{F}} \overline{\mathbf{P}} \cdot \nabla_{X} N^{l} \mathrm{~d} V
\end{aligned}
$$

And finally, to close up the set-up of the non-linear finite element procedure, the updating for the global unknowns increments $\rho_{I}$ and $\varphi_{J}$ are

$$
\begin{array}{ll}
\Delta \rho_{I}=\Delta \rho_{I}+\mathrm{d} \rho_{I} & \forall \quad I \text { in } \mathbb{B} \\
\Delta \varphi_{J}=\Delta \varphi_{J}+\mathrm{d} \varphi_{J} & \forall \quad J \quad \text { in } \mathbb{B}
\end{array}
$$

can be expressed in terms of the solution of the linearized system of equations (??).

Remark 4 (Different times scales in biological tissues) The biological and the mechanical phenomena in biological tissue have usually different time scales. The balance of linear momentum can be decoupled, considering the mechanical part, which usually is presented in a larger time scale, as 
quasi-static. The quasi-static process is recovered by neglecting the dynamic contributions presented in $N_{\varphi}^{j} \rho_{0}\left[\boldsymbol{p}_{n+1}-\boldsymbol{p}_{n}\right] / \Delta t$ of equation (??) $)_{2}$ and the $N^{j} \rho_{0} / \Delta t \boldsymbol{I} N^{l}$ term of equation (??) $)_{4}$

Remark 5 (Different material scales in biological tissues) Also, in biological tissue the magnitudes of different properties, as mechanical or biological properties differ from several orders of magnitude. It is usually common to present a normalized, e.g. a relative density $\left[\rho_{0} / \rho_{0}^{*}\right]$ in terms of the initial density $\rho_{0}^{*}$, and introduce it as the primary unknown in order to avoid ill-conditioned matrices.

3.6 Numerical implementation of volumetric growth

The numerical implementationof the volumetric growth is within the kinematic part of the formulation rather than in the coupling of the finite element formulation. The evolution of growth is integrated in time using an implicit Euler backward scheme,

$$
\dot{\vartheta}=\left[\vartheta^{\mathrm{n}+1}-\vartheta^{\mathrm{n}}\right] / \Delta t
$$

where $\Delta t$ denotes the current time increment. This allows us to introduce the discrete local residual

$$
\mathrm{R}=\vartheta^{\mathrm{n}+1}-\vartheta^{\mathrm{n}}-\kappa(\vartheta) \phi(\boldsymbol{\Xi}) \Delta t
$$

To solve this non-linear equation, the residual is expanded up to the first order term which allows us to solve the problem, again, within a Newton iterative scheme according to the following equation,

$$
\mathrm{R}(\vartheta)^{\mathrm{n}}+\left.\partial_{\vartheta} \mathrm{R}(\vartheta)^{\mathrm{n}}\right|_{\vartheta^{\mathrm{n}}}\left[\vartheta^{\mathrm{n}+1}-\vartheta^{\mathrm{n}}\right] \doteq 0
$$

The tangent of the residual

$$
\mathcal{K}=\mathrm{d}_{\vartheta} \mathrm{R}=1-\left[\kappa \partial_{\vartheta} \phi+\vartheta \partial_{\vartheta} \kappa\right] \Delta t
$$

allows us to incrementally update the growth multiplier $\vartheta^{\mathrm{n}+1} \leftarrow \vartheta^{\mathrm{n}}-\mathrm{R} / \mathcal{K}$, Table ?? summarizes the local algorithmic treatment of the numerical procedure, which is embedded in the finite element framework on the constitutive level. 
Table 1: Algorithm for implicit Euler scheme of strain-driven volumetric growth

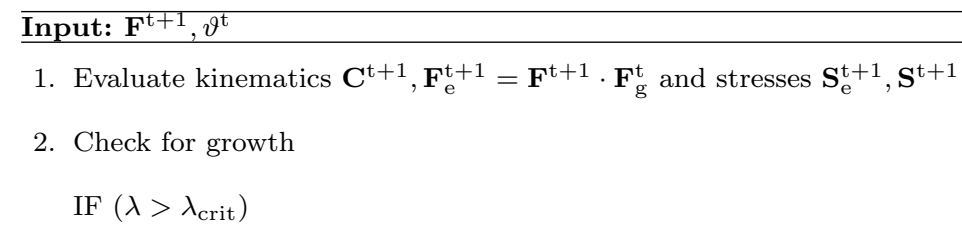

THEN Determine new growth multiplier

WHILE R $>$ tol DO

Calculate residual $\mathrm{R}=\vartheta^{\mathrm{n}+1}-\vartheta^{\mathrm{n}}-\kappa(\vartheta) \phi(\boldsymbol{\Xi}) \Delta t$

Calculate tangent $\mathcal{K}=1-\left[\kappa \partial_{\vartheta} \phi+\vartheta \partial_{\vartheta} \kappa\right] \Delta t$

Update growth $\quad \vartheta^{\mathrm{n}+1} \leftarrow \vartheta^{\mathrm{n}}-\mathrm{R} / \mathcal{K}^{-1}$

END

3. Compute Cauchy stresses $\boldsymbol{\sigma}^{\mathrm{t}+1}$

Compute tangent moduli $\mathbf{c}^{\mathrm{t}+1}$

Output: $\boldsymbol{\sigma}^{\mathrm{t}+1}, \mathbf{c}^{\mathrm{t}+1}, \vartheta^{\mathrm{n}+1}$

Remark 6 (Stress-driven anisotropic growth) The evolution of the growth multiplier $\vartheta$ can be expressed in terms of a stress-based stimulus ??,

$$
\dot{\vartheta}=\kappa(\vartheta) \phi\left(\mathbf{M}_{\mathrm{e}}\right)
$$

where

$$
\phi\left(\mathbf{M}_{\mathrm{e}}\right)=\operatorname{tr}\left(\mathbf{M}_{\mathrm{e}}\right)-\mathrm{M}_{\mathrm{crit}}
$$

Here, $\mathbf{M}_{\mathrm{e}}=\mathbf{C}_{\mathrm{e}} \cdot \mathbf{S}_{\mathrm{e}}$ is the elastic Mandel stress and $\mathbf{M}_{\mathrm{crit}}$ denotes the physiological pressure above which growth occurs. This quantity id usually used due to thermodynamic aspetcs as it represents the conjugate variable of the pull-back of 1 . The derivative of the growth criterion with respect to the growth multiplier $\vartheta$ required for the consistent linearization reads

$$
\partial_{\vartheta} \phi=-\partial_{\vartheta} \mathbf{C}_{\mathrm{e}}: \mathbf{S}_{\mathrm{e}}+\mathbf{C}_{\mathrm{e}}: \partial_{\vartheta} \mathbf{S}_{\mathrm{e}}
$$

with $\partial_{\vartheta} \mathbf{C}_{\mathrm{e}}=-\mathbf{F}_{\mathrm{g}}^{-\mathrm{t}} \cdot \partial_{\vartheta} \mathbf{F}_{\mathrm{g}}^{\mathrm{t}} \cdot \mathbf{C}_{\mathrm{e}}-\mathbf{C}_{\mathrm{e}} \cdot \partial_{\vartheta} \mathbf{F}_{\mathrm{g}} \cdot \mathbf{F}_{\mathrm{g}}^{-1}$ and $\partial_{\vartheta} \mathbf{S}_{\mathrm{e}}=\frac{1}{2} \mathbf{C}_{\mathrm{e}}: \partial_{\vartheta} \mathbf{C}_{\mathrm{e}}$. Finally, to complete the tangent moduli in (??), we provide the derivative of the growth multiplier with respect to the Cauchy-Green strain tensor, $\partial_{\mathbf{C}} \vartheta=\partial_{\mathbf{C e}} \vartheta: \partial_{\mathbf{C}} \mathbf{C}_{\mathrm{e}}=\kappa \Delta t\left[\frac{1}{2} \mathbf{C}_{\mathrm{e}}: \mathbf{C}_{\mathrm{e}}+\mathbf{S}_{\mathrm{e}}\right] / \mathcal{K}$ 
Remark 7 (Strain-driven anisotropic growth) Alternatively, we can define the evolution of the growth multiplier $\vartheta$ in terms of a strain-based stimulus?,

$$
\dot{\vartheta}=\kappa(\vartheta) \phi\left(\mathbf{F}_{\mathrm{e}}\right)
$$

using a strain-driven growth criterion

$$
\phi\left(\mathbf{F}_{\mathrm{e}}\right)=\lambda_{\mathrm{e}}-\lambda_{\text {crit }}=\mathbf{F}_{\mathrm{e}}:[\mathbf{n} \otimes \mathbf{n}]-\lambda_{\text {crit }} .
$$

Here $\lambda_{\text {crit }}$ denotes a physiological stretch above which growth occurs. $\lambda_{\mathrm{e}}=\mathbf{F}_{\mathrm{e}}:[\mathbf{n} \otimes \mathbf{n}]$ is the strecth in the direction of the line element $\mathbf{n}$. The remaining term for the tangent moduli then simply reads

$$
\partial_{\vartheta} \phi=-\lambda / \lambda_{\mathrm{g}}^{2}
$$

Remark 8 (Phenomenological growth) In the simplest case, when $\vartheta$ do not depend on mechanical variables and is directly imposed as

$$
\dot{\vartheta}=\kappa(\vartheta) \phi
$$

so that there are not dependencies on the mechanical variables.

\section{Biological examples: Density growth}

In this section we are going to show some examples of literature where the theory and numerical formulation described previously is used, for, e.g study the collagen turnover in hypertension disease or wound healing.

\subsection{Remodeling during hypertension}

One of the biological processes at which remodeling take place is the collagen turnover during hypertension. Although collagen synthesis and removal has been treated in literature driven by mechanical quantities, there are not many models considering the actual substance that make collagen to remodel. Here we summarize a computational model by ? studying some of these 
aspects. The model consider the synthesis and degradation of TGF- $\beta$, MMP and TIMP, which are the most relevant substances in collagen remodeling, based on the description of ?. The material density is allowed to evolve in time according to the mass balance for open system thermodynamics (see Eq. ??), and adopt a source term, $\mathcal{R}$, similar to that described by ??, as

$$
\dot{\rho}=\mathcal{R} \quad \text { with } \quad \mathcal{R}=\left[\frac{\rho}{\rho^{*}}\right]^{-m} \lambda-\lambda^{*},
$$

where the exponent $m$ typically varies between two and three, $\lambda^{*}$ is the stretch of the homeostatic equilibrium state and $\lambda=[\mathbf{r} \cdot \mathbf{C} \cdot \mathbf{r}]^{1 / 2}$ is the stretch of a fiber with orientation $\mathbf{r}$. $\rho$ is the current density of the substance at hand, $\rho^{*}$ is the initial density and $\dot{\rho}$ the material time derivative of $\rho$.

Depending on the stretch of the line element, which it is understood to be the main driving force for these processes, the substances density will increase or decrease. The stretch increases change the growth factors density, TGF- $\beta$, TIMP or MMPs, as

$$
\begin{gathered}
\mathcal{R}_{\mathrm{TGF}-\beta}=\dot{\rho}_{\mathrm{TGF}-\beta}=\gamma_{\mathrm{TGF}-\beta}\left[\left[\frac{\rho_{\mathrm{TGF}-\beta}}{\rho_{\mathrm{TGF}-\beta}^{*}}\right]^{-m_{\mathrm{TGF}-\beta}} \lambda_{\mathrm{e}}-\lambda^{*}\right] . \\
\mathcal{R}_{\mathrm{TIMP}}=\dot{\rho}_{\mathrm{TIMP}}=\gamma_{\mathrm{TIMP}}\left[\left[\frac{\rho_{\mathrm{TIMP}}}{\rho_{\mathrm{TIMP}}^{*}}\right]^{-m_{\mathrm{TIMP}}} \lambda_{\mathrm{e}}-\lambda^{*}\right] .
\end{gathered}
$$

and

$$
\mathcal{R}_{\mathrm{MMP}}=\gamma_{\mathrm{MMP}} \mathcal{R}_{\mathrm{TIMP}}
$$

This implies that the substance will be upregulated, for blood pressures above a characteristic threshold level, if $\lambda_{\mathrm{e}}>\lambda^{*}$, down regulated for blood pressures below if $\lambda_{\mathrm{e}}<\lambda^{*}$ and otherwise remain constant. $\gamma$ and $m$ are parameters that govern the evolution of the process.

As described in the previous section, the non-linear differential equations of collagen deposition and absorption ( Eqs. ?? and ??) was solved by a standard Euler backward scheme,

$$
\dot{\rho}_{0}=\left[\rho_{0}^{j+1}-\rho_{0}^{j}\right] / \Delta t
$$

for given initial conditions $\left.\rho_{0}\right|_{t_{0}=0}=\rho_{0}^{*}$. 
The mass transport problem of the substances described above was computed (??) as described previously. The flux term, as usually in biological tissue, was given as

$$
\mathbf{J}=-\mathrm{D}_{e f f} \nabla \rho_{0}+\rho_{0} \mathrm{D}_{t} \mathbf{u}
$$

representing the diffusive contribution, driven by density gradients, and the convective contribution, controlled by pressure gradients with $\mathbf{u}$ being the displacement vector, usually described in biological tissue by means of the Darcy's law.

Finally, the collagen turnover was described

$$
\hat{\mathcal{R}}_{\mathrm{col}}=\gamma_{\mathrm{dep}} \hat{\mathcal{R}}_{\mathrm{TGF}-\beta}+\gamma_{\mathrm{abs}} \hat{\mathcal{R}}_{\mathrm{MMP}}
$$

$\hat{\mathcal{R}}_{\mathrm{TGF}-\beta}$ and $\hat{\mathcal{R}}_{\mathrm{MMP}}$ are actual amount of TGF- $\beta$ and MMP at every integration point after the mass transport phenomena. $\gamma_{\text {dep }}, \gamma_{\text {abs }} \in \mathbb{R}^{+}$denote the sensitivities of collagen deposition and absorption in response to changes in TGF- $\beta$ and MMP, respectively. These set of equations can be solved numerically by applying a local Newton-Raphson iteration. Table ?? summarizes the algorithm to compute the update of the local collagen content.

Based on these procedure ? presented the results of mass production, transport and later collagen remodeling in hypertensive cases. In Fig. ?? and ?? we show some results of the TGF- $\beta$ and MMP production in a carotid artery model.

4.2 Wound healing

Wound healing have been also widely studied computationally. Some of the latest works included finite element frameworks, constituents turnover, collagen synthesis and wound contraction. The idea behind many of these models follow the description presented above.

For example, ? introduce the problem in terms of a chemical signal, inflammation, fibroblasts and a isotropic hyperelastic mechanical model. They described a coupled formulation, as described 
Table 2: Algorithm to compute the local collagen content using an implicit Euler backward scheme within a finite element framework.

\section{Input: $\mathbf{F}^{j+1}$, internal variables at time $j$}

1. Evaluate kinematics

$\mathbf{C}^{j+1}$ and constitutive equations $\Psi^{j+1}, \mathbf{S}^{j+1}$

2. Check stretch

IF $\left(\lambda_{\mathrm{e}}^{j+1} \neq \lambda^{*}\right)$ THEN GO TO III

ELSE GO TO IV

3. Local Newton iteration. WHILE $\mathrm{R}_{\rho_{\mathrm{TGF}-\beta}}>$ tol or $\mathrm{R}_{\rho_{\mathrm{TIMP}}}>$ tol

Calculate local TGF- $\beta$ and TIMP residuals

$$
\begin{aligned}
& \mathrm{R}_{\mathrm{TGF}-\beta}=-\rho_{\mathrm{TGF}-\beta}^{j+1}+\rho_{\mathrm{TGF}-\beta}^{j}+\mathcal{R}_{\mathrm{TGF}-\beta} \Delta t \doteq 0 \\
& \mathrm{R}_{\mathrm{TIMP}}=-\rho_{\mathrm{TIMP}}^{j+1}+\rho_{\mathrm{TIMP}}^{j}+\mathcal{R}_{\mathrm{TIMP}} \Delta t \doteq 0
\end{aligned}
$$

Calculate local TGF- $\beta$ and TIMP tangents

$$
\begin{aligned}
& \mathrm{K}_{\mathrm{TGF}-\beta}=1+\frac{m_{\mathrm{TGF}-\beta}}{\rho_{\mathrm{TGF}-\beta}^{k}}\left[\frac{\rho_{\mathrm{TGF}-\beta}^{k}}{\rho_{\mathrm{TGF}-\beta}^{*}}\right]^{-m_{\mathrm{TGF}-\beta}} \Psi_{\mathrm{e}}\left(\overline{I_{4}}\right) \Delta t \\
& \mathrm{~K}_{\mathrm{TIMP}}=1+\frac{m_{\mathrm{TIMP}}}{\rho_{\mathrm{TIMP}}^{k}}\left[\frac{\rho_{\mathrm{TIMP}}^{k}}{\rho_{\mathrm{TIMP}}^{*}}\right] \begin{array}{c}
\mathrm{TIMP} \\
\Psi_{\mathrm{e}}\left(\overline{I_{4}}\right) \Delta t
\end{array}
\end{aligned}
$$

Update TGF- $\beta$ and TIMP concentrations

$$
\begin{aligned}
& \rho_{\mathrm{TGF}-\beta}^{\mathrm{j}+1} \leftarrow \rho_{\mathrm{TGF}-\beta}^{\mathrm{j}+1}-\mathrm{R}_{\rho_{\mathrm{TGF}-\beta}} / \mathrm{K}_{\rho_{\mathrm{TGF}-\beta}} \\
& \rho_{\mathrm{TIMP}}^{\mathrm{j}+1} \leftarrow \rho_{\mathrm{TIMP}}^{\mathrm{j}+1}-\mathrm{R}_{\rho_{\mathrm{TIMP}}} / \mathrm{K}_{\rho_{\mathrm{TIMP}}}
\end{aligned}
$$

4. Update MMP concentration and collagen content

$$
\begin{aligned}
& \rho_{\mathrm{MMP}}^{j+1}=\gamma_{\mathrm{MMP}} \rho_{\mathrm{TIMP}}^{j+1} \\
& \rho_{\mathrm{Col}}^{j+1}=\gamma_{\mathrm{dep}} \rho_{\mathrm{TGF}-\beta}^{j+1}+\gamma_{\mathrm{abs}} \rho_{\mathrm{MMP}}^{j+1}
\end{aligned}
$$

5. Calculate stresses $\mathbf{S}^{t+1}$ 


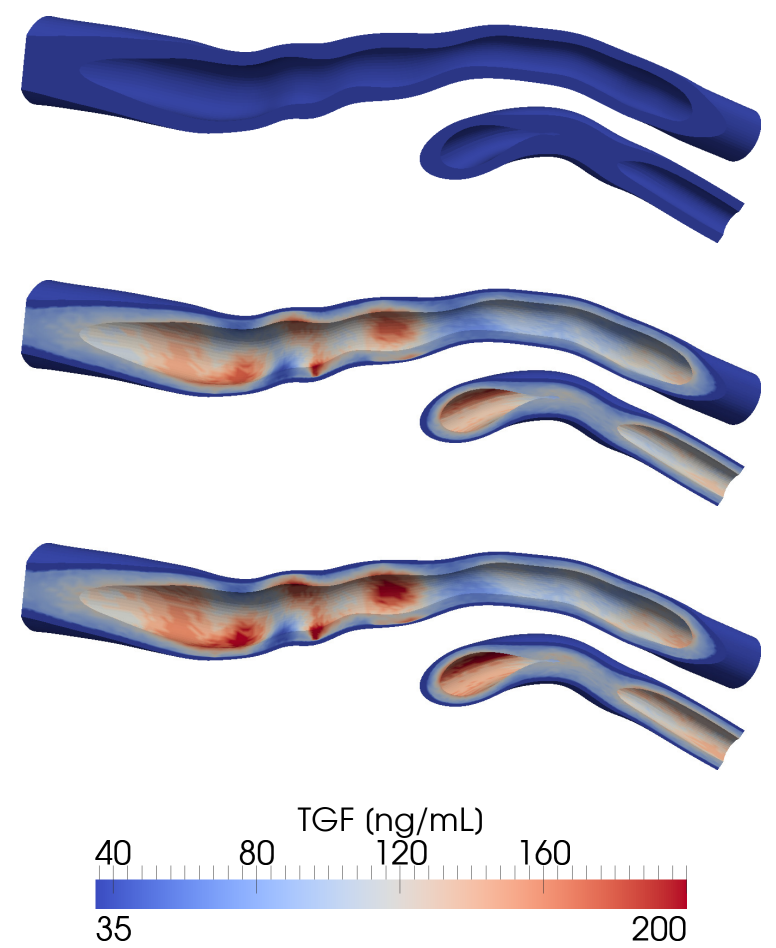

Fig. 3: Evolution of the mass production of TGF- $\beta$ due to SMC activity in different longitudinal cuts.

in the previous section, of the cross-talk between chemical, biological, and mechanical signals. They looked at how different wound shapes modify the effect of healing and how biological substances and mechanical properties changes over time (see Fig. ??). They considered a chemical model, characterized by the inflammation process governed by a isotropic diffusion flux term and a degradation assumption for the source term. And a so-called biological approach governed by the fibroblasts, where the flux reflect the isotropic diffusion due to the own fibroblast gradient concentration and the inflammation concentration and the source term also taking into account the inflammation process. The mechanical balance law couple the effect of both the chemical and biological mass balance laws. These set of equations posses a three-coupled balance equations that were implicitly solved in the temporal and spatial domain as described in the previous section. 


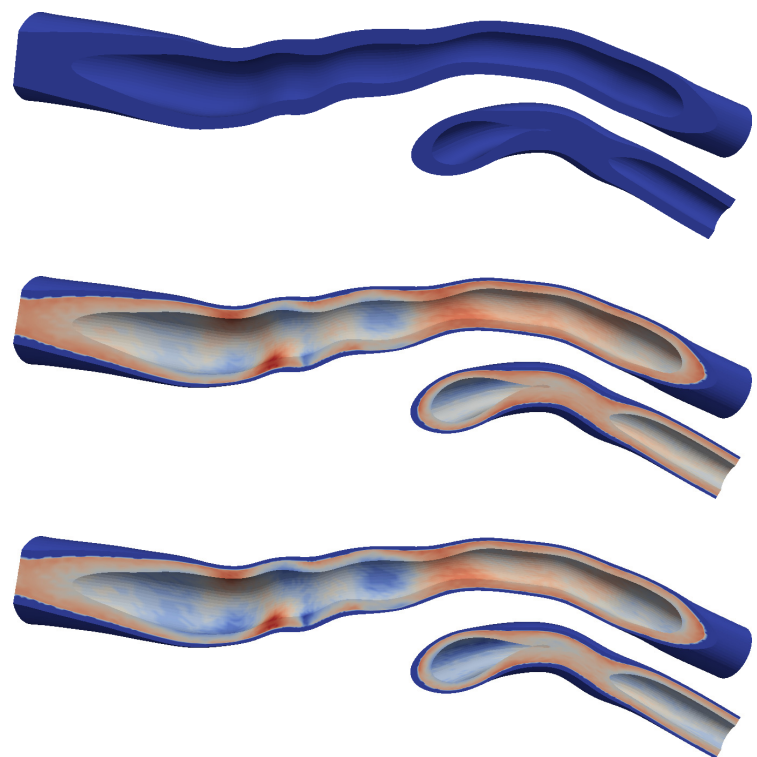

$26 \quad 28^{\mathrm{MMP}(\mathrm{ng} / \mathrm{mL})} 30_{32} \quad 34$

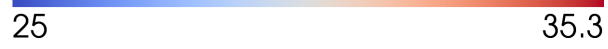

Fig. 4: Evolution of the mass production of MMP-1 due to SMC activity in different longitudinal cuts.

In a very similar fashion, ? described the regulation of the healing process by the cells through a mechano-sensing mechanism of the contraction that the cells exert. They adjust the contraction level to determine the tissue behavior that regulate all main activities in wound healing, e.g. proliferation, differentiation and matrix production. So there is not only a turnover of substances but also a feedback loop that makes the wound healing to change as the self wound evolves. They computed, e.g., the amount of myofibroblast and collagen in the tissue, as shown in Fig. ??

\section{Biological examples: Volumetric growth}

The computational mechanical growth model described in section ?? have been used to simulate different process in biological tissue as, e.g., the thickening in hypertension in arteries (??), the 


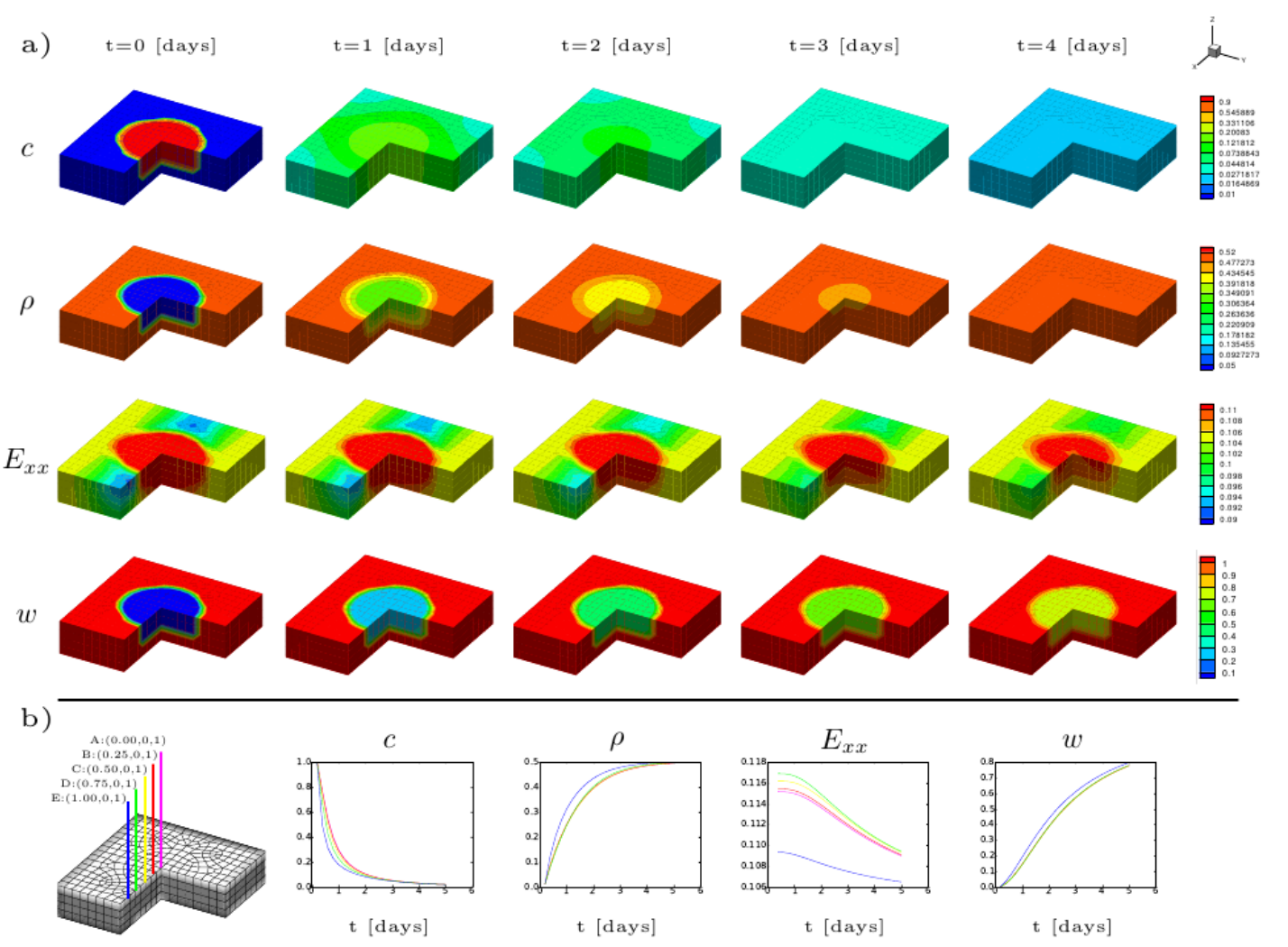

Fig. 5: Wound healing evolution over time of inflammatory signal, fibroblast density, GreenLagrange strain and collagen content (from ??, with permission).

heart (?),the brain (??) and skin (??) among others. Herein, we will show some examples of artery thickening and brain folding.

\subsection{Brain folding}

Several studies have used the multiplicative decomposition of the deformation gradient (see Section ??), and its numerical implementation in a finite element framework. This approach has been used for the study of brain development and folding, one of the most intriguing question in developmental biology. Brain folding is believed to be controlled by the mechanical microenvironment, where the microstrucuture change due to the mechanical loading. Besides, brain folding is directly related to 

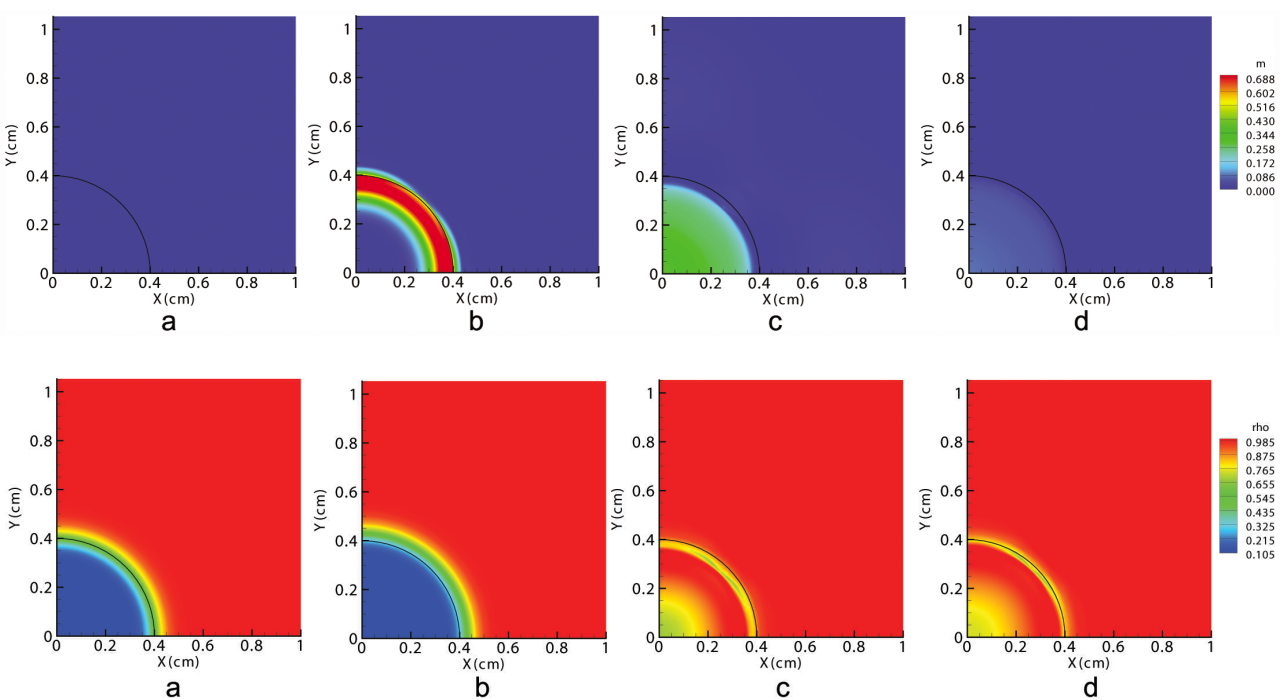

Fig. 6: Top and bottom, myofibroblasts and collagen distributions over time, respectively. The black line denotes the edge between the initial wound and the surrounding skin. Reprinted from ?.

several diseases. Here we show some results from ? and ? of how they predicted the shape of the brain folding by a bifurcation and a growth description (see Fig. ??(a) and ??(b)). ? expressed the growth of the cortical layer phenomenologically as $\dot{\theta}=\mathrm{G}_{c}$ while the subcortical tissue growth was strain induced as $\dot{\theta}=\mathrm{G}_{c}\left[\mathrm{~J}^{e}-\mathrm{J}^{0}\right]$. They motived that the axon elongation was the one that lead to volume expansion. ? also looked at brain folding with similar assumption but considering a stress-based approach. They looked at effects of cortical growth rate caused by tangential growth of the cortical layer and the growth of the subcortical growth due to stresses arising.

\subsection{Arterial thickening during hypertension}

To account for experimental observations of SMC growth (?), ? defined the growth tensor as

$$
\mathbf{F}_{\mathrm{g}}=\mathbf{I}+[\vartheta-1] \mathbf{n}_{\mathrm{r}} \otimes \mathbf{n}_{\mathrm{r}}
$$

where $\vartheta$ is the scalar-valued growth multiplier that defines the level of growth and $\mathbf{n}_{\mathrm{r}}$ characterizes the radial direction ?. The mechanical stimulus that triggers growth is the stretch $\lambda_{\mathrm{e}}=\mathbf{F}_{\mathrm{e}}:\left[\mathbf{n}_{\mathrm{z}} \otimes \mathbf{n}_{\mathrm{z}}\right]$, 

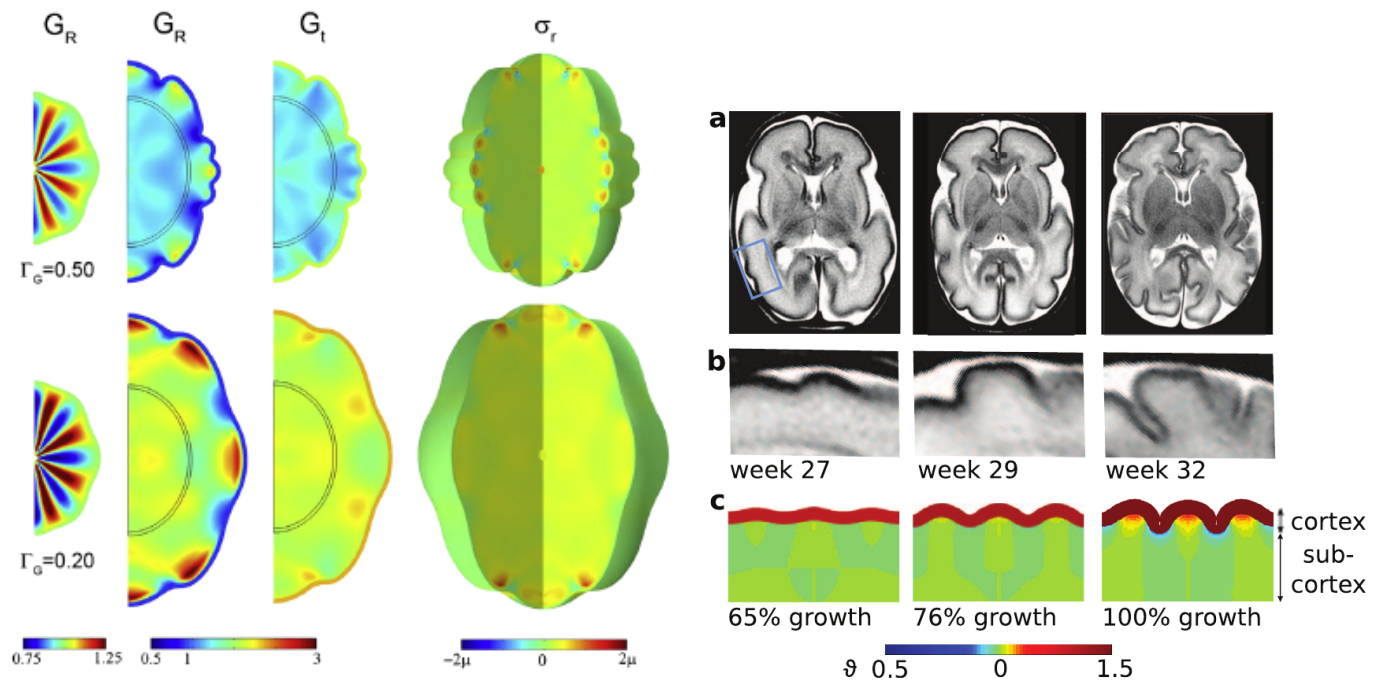

(a) Growth patters and stresses due to different com- (b) Brain patter formation from phenomenological binations of different relative growth rates (?). and strain-based models (?).

Fig. 7: Folding patter formation of the brain simulated with the finite element method. On the left results from ? (with permission) from stress-based approaches and right results from ? (with permission) from strain motivated model and bifurcation theory.

and the growth criterion is expressed as $\phi\left(\mathbf{F}_{\mathrm{e}}\right)=\lambda_{\mathrm{SMC}}-\lambda_{\text {crit }}$. This particular format of the growth tensor characterizes smooth muscle cell thickening in the radial direction $\mathbf{n}_{\mathrm{r}}$.

? described the spatio-temporal evolution of growth in a human carotid artery model. Figures ?? shows the evolution of the growth multiplier and the maximum principal stresses in the adventitia and the medial layer in a slice of the common carotid artery at four different time steps. The results were in agreement with the smooth variations in stretch between the normotensive and the hypertensive states. While stresses in the adventitia increase the stresses in the media decrease. The increase was due to the expansion of the media layer while compression is due to the constrain of the adventitia to the expansion. 


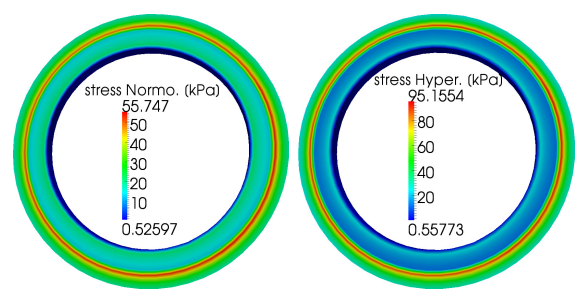

(a) Stress in nor- (b) Stress in hyper-

motensive state. tensive state.

$\vartheta[-]$

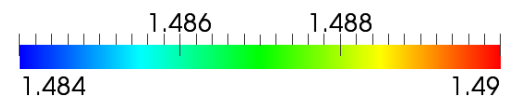

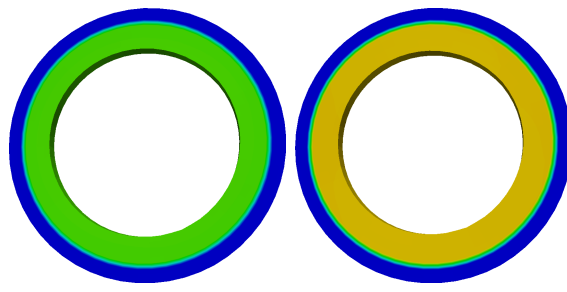

(d) Time $t=5$ days

(e) Time $t=15$ days
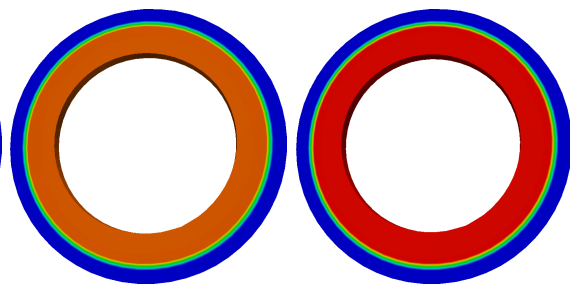

(f) Time $t=40$ days

(g) Time $\mathrm{t}=100$ days
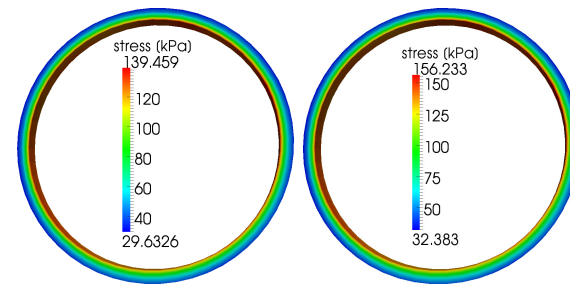

(i) Time $t=15$ days
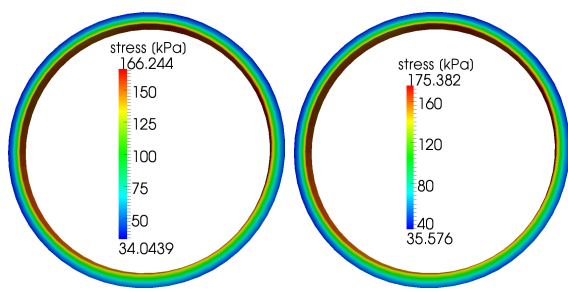

(h) Time $\mathrm{t}=5$ days

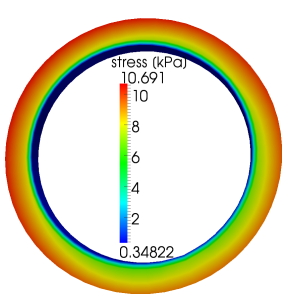

(1) Time $t=5$ days

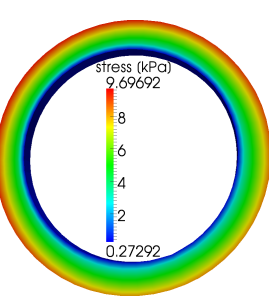

(m) Time $\mathrm{t}=15$ days (j) Time $t=40$ days

(k) Time $\mathrm{t}=100$ days

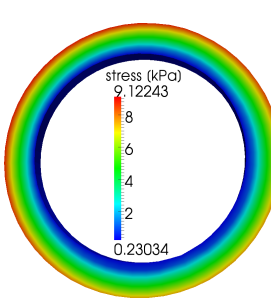

(n) Time $\mathrm{t}=40$ days

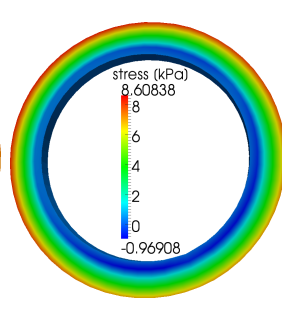

(o) Time $\mathrm{t}=100$ days

Fig. 8: Spatio-temporal evolution of growth in a representative slice of the common carotid artery. Growth multiplier (top) maximum principal stresses in the adventitia (middle) and maximum principal stresses in the media (bottom) at 5, 15, 40, and 100 days of hypertension (with permission). 


\section{Biological examples: Cell remodeling}

Biological remodeling can occur in any kind of biological tissue and many of these changes can lead to further adaptation processes, as the formation of atherome plaque (?). In terms of cellular models capturing these features not much have been done. ?? studied the orientation of the preferential direction of the cell, where the reorientation is assumed to be controlled by the matrix behavior and the forces that arise from the active regulation of the cell in a dipole-like manner. In terms of the internal components of the cell, some simulations have been also performed within molecular and Brownian dynamics of filaments and network actin filaments??.

Recently, ? developed a model that brought together some approaches reported in the literature for remodeling of single fibers (??) or more complex structures (?). They took into account the reorientation of the mean direction of a given fibered structure and the reorientation of the individual fibrils or filaments were recovered by means of a micro-sphere based approach. The microspherebased approach constitutes an homogenization technique that has been used previously for brittle materials (?), damage and fracture (???), polymers (?) and biological tissue (???), among many other applications. The homogenization or continuous averaging $\langle(\bullet)\rangle$ of a given variable $(\bullet)$ is carried out by integrating over the unit sphere surface. In order to perform a numerical implementation, the integral is computed by summing up the integrand over $m$ discrete orientation vectors with the corresponding weight factors $w^{i}$ as

$$
\langle(\bullet)\rangle=\frac{1}{4 \pi} \int_{\mathbb{U}^{2}}(\bullet) \mathrm{dA} \approx \sum_{i=1}^{m} w^{i}(\bullet)_{i},
$$

where $\mathrm{dA}$ is the differential area element of the unit sphere that may be written in terms of the spherical angles $\alpha \in[0, \pi)$ and $\phi \in[0,2 \pi)$ as $\mathrm{dA}=\sin (\alpha) \mathrm{d} \phi \mathrm{d} \alpha$. See ? for a study of the numerical performance of the integration scheme.

The SEDF contribution of the microstructure to the macroscopic effect may be homogenized

as

$$
\Psi_{\mathrm{ani}}=\frac{1}{4 \pi} \int_{\mathbb{U}^{2}} \rho \psi(\bar{\lambda}) d A \approx \sum_{i=1}^{m} \rho_{i} w^{i} \psi\left(\bar{\lambda}_{i}\right)
$$


At the microscale the elements can be described, e.g., by the well-established Worm-Like Chain model (?). Statistical distributions can also provide more flexibility in modeling the dispersion of the fibrils (??).

The realigment of the microsphere was described extending the model of ?? for single line elements and ? in the context of the micro-sphere. The realignment depends on the maximum principal direction of a driving stimulus $\boldsymbol{\Xi}$, such that $\mathbf{r}_{i} \longmapsto \boldsymbol{\Xi}_{3}$, with $I=1,2,3$ the eigenvectors associated to the minimum, medium and maximum eigenvalues of $\boldsymbol{\Xi}$. This leads to $\boldsymbol{\omega}_{i}:=\mathbf{r}_{i} \times \boldsymbol{\Xi}_{3}$ where $\boldsymbol{\omega}_{i}$ and $\mathbf{r}_{i}$ are the angular velocity and the unit vector of each integration direction. The realignment of a given general second order tensor, $\mathbf{Q} \in \mathrm{SO}(3)$ was defined by the eigenvectors of the driving stimulus $\boldsymbol{\Xi}$. The rotation tensor, $\mathbf{R}=\boldsymbol{\Xi} \cdot \mathbf{Q}^{-1} \in \mathrm{SO}(3)$ was used to define a geometrically exact update in terms of the exponential mapping as $\mathbf{R}=\exp (-\boldsymbol{\varepsilon} \cdot \boldsymbol{\omega})$ with $\boldsymbol{\varepsilon}$ denotes the third-order permutation symbol. In short, it is possible to define a rotation vector $\boldsymbol{\omega}$, through which the initial base rotate to the final position(see ? for details).

From this approach, the rate of $\mathbf{Q}$ is given by

$$
\dot{\mathbf{Q}}=\hat{\mathbf{n}}^{\boldsymbol{\omega}} \cdot \mathbf{Q} \text {. }
$$

where $\mathbf{n}^{\boldsymbol{\omega}}=\boldsymbol{\omega} / \boldsymbol{\omega}, \boldsymbol{\omega}=\|\boldsymbol{\omega}\|$ and $\hat{\mathbf{n}}^{\boldsymbol{\omega}}=-\boldsymbol{\varepsilon} \cdot \mathbf{n}^{\boldsymbol{\omega}}$ are the norm of the angular velocity, its unit direction and the so called hat map of $\boldsymbol{\omega}$, respectively. And for $\mathbf{r}_{i}$ evolution rate reads

$$
\dot{\mathbf{r}_{i}}=\left[\mathbf{I}-\mathbf{r}_{i} \otimes \mathbf{r}_{i}\right] \cdot \boldsymbol{\Xi}_{3}
$$

For the numerical approach, and similarly to previous sections, a time interval $T$ and a number of subintervals, $n, T=\bigcup_{0}^{n-1}\left[t^{n}, t^{n+1}\right]$ was considered where the time increment is given by $\Delta t=$ $t^{n+1}-t^{n}$. The evolution of the base and the $\mathbf{r}_{i}$ at time $n$ will evolve in the exponential mapping context as

$$
\begin{gathered}
\mathbf{Q}^{n+1}=\exp (-\varepsilon \cdot \boldsymbol{\omega} \Delta t) \cdot \mathbf{Q}^{n} \text { and } \\
\mathbf{r}_{i}^{n+1}=\exp \left(\hat{\mathbf{n}}_{i}^{\boldsymbol{\omega}} \Delta t\right) \cdot \mathbf{r}_{i}^{n}
\end{gathered}
$$


Table 3: Algorithm to compute remodeling of cell-like structures.

\section{Input: $\mathbf{F}^{j+1}$, internal variables at time $j$}

I Evaluate kinematics $\mathbf{C}^{j+1}$ and constitutive equations $\Psi^{j+1}, \mathbf{S}^{j+1}$ and $\mathbf{M}^{j+1}$

II Compute stimulus tensor $\boldsymbol{\Xi}$

III Update orientation variables.

Evaluate exponential mapping for the evolution of $\mathbf{Q}^{j+1}$ and $\mathbf{r}_{i}^{j+1}$

$$
\begin{aligned}
\mathbf{Q}^{j+1} & =\exp \left(\hat{\mathbf{n}}^{\boldsymbol{\omega}} \Delta t\right) \cdot \mathbf{Q}^{j} \\
\mathbf{r}^{j+1} & =\exp \left(\hat{\mathbf{n}}^{\boldsymbol{\omega}} \Delta t\right) \cdot \mathbf{r}^{j}
\end{aligned}
$$

IV Calculate stresses $\boldsymbol{\tau}^{j+1}$ and tangent operator related to the Jaumann rate as

$$
\begin{aligned}
& \boldsymbol{\tau}^{j+1} \approx 2 \mathbf{b}^{j+1} \cdot\left(C_{1}-C_{2} I_{1}^{j+1}\right)+\sum_{i=1}^{m} \rho_{i} \overline{\boldsymbol{\tau}}_{i}^{j+1} w^{i} \approx \sum_{i=1}^{m}\left[\rho_{i} \psi^{\prime}\left(\bar{\lambda}_{i}\right) \bar{\lambda}_{i}^{-1} \overline{\mathbf{t}}_{i}^{j+1} \otimes \overline{\mathbf{t}}_{i}^{j+1}\right] w^{i} \\
& \overrightarrow{\mathbf{c}}^{j+1}=\left[\mathbf{c}^{j+1}+1 / 2\left[\delta \tau^{j+1}+\tau^{j+1} \delta+\delta \tau^{j+1}+\tau^{j+1} \delta\right]\right] / J \text { with } \\
& \mathbf{c}^{j+1} \approx \sum_{i=1}^{m} n \rho_{i} w_{i}\left[\psi_{i}^{\prime \prime}{ }^{j+1}-\psi_{i}^{\prime}{ }^{j+1} \bar{\lambda}^{-1}\right] \bar{\lambda}^{-2} \overline{\mathbf{t}}_{i}^{j+1} \otimes \overline{\mathbf{t}}_{i}^{j+1} \otimes \overline{\mathbf{t}}_{i}^{j+1} \otimes \overline{\mathbf{t}}_{i}^{j+1}
\end{aligned}
$$

Output: $\boldsymbol{\tau}^{j+1}, \nabla^{j+1}, \mathbf{Q}^{j+1}, \mathbf{r}^{j+1}$

This approach follows an explicit updating scheme, with dependencies on the current position, different from the implicit scheme in ? where the updated quantities were obtained by means of a Newton iteration scheme. However, this explicit approach is a reasonable approach for the gradual realignment of biological tissues. We have outlined the main steps of the algorithmic scheme in Table ??

Remark 9 In order to compute the exponential map, it will be rewritten (see e.g. ?) by the Rodrigues formula, given $\mathbf{R}(\boldsymbol{\omega})$, as

$$
\exp \left(\hat{\mathbf{n}}^{\boldsymbol{\omega}} t\right)=[\sin (\|\boldsymbol{\omega}\| t)] \hat{\mathbf{n}}^{\boldsymbol{\omega}}+[1-\cos (\|\boldsymbol{\omega}\| t)] \mathbf{n}^{\boldsymbol{\omega}} \otimes \mathbf{n}^{\boldsymbol{\omega}}+\cos (\|\boldsymbol{\omega}\| t) \mathbf{I},
$$

Following the above approach but from a implicit algorithm point of view, ? studied how the vectors defining the anisotropy of the material reorient due to different load states (see Fig. ??). ? described the evolution of the micro-structural orientation and the changes in the stress distribution in a rectangular plate due to changes in the fibered structures (see Fig ??), which evolve based on 

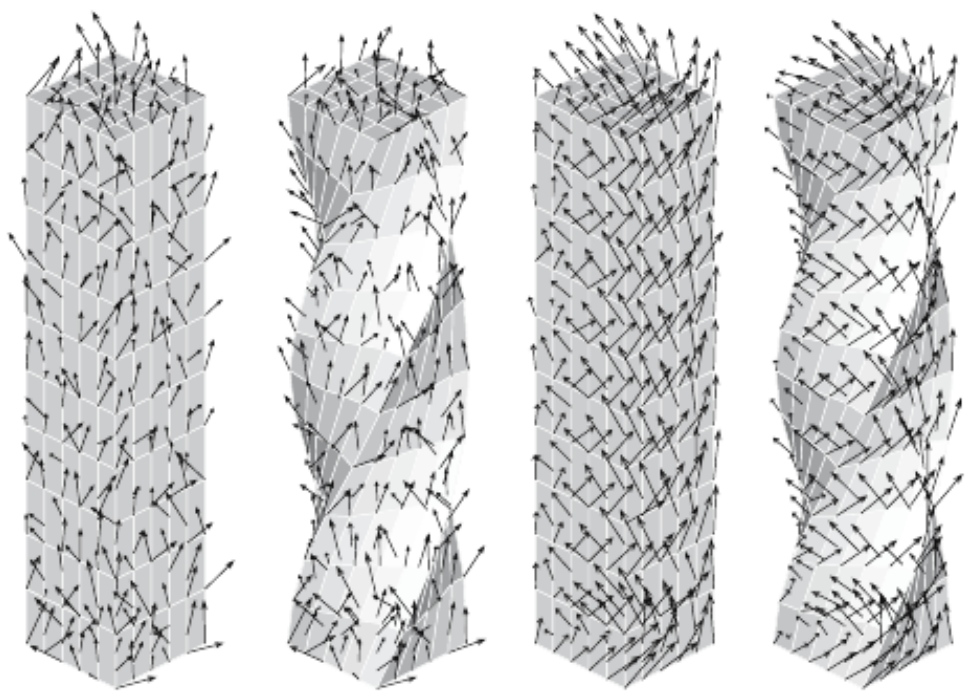

Fig. 9: Evolution of the fibers direction when applying torsion to a square rod in the reference and deformed configuration at different time steps (reprinted from ? with permission).

mechanical variables, as strain or stress magnitudes. It showed a decrease of stresses due to the stiffening of the material and the fixed applied force and the progressively alignment of the cell in the direction of the load. Following this approach they also described energetic evolution and include it in a finite element model presenting a biological example of the model discussed above. These concepts can be extrapolated to study the cell reorientation discussed in the introductory section.

\section{Discussion}

7.1 Current achievements in biological continuum models

During this review on the continuum approach of growth and remodeling we have been through different biological examples where adaptive phenomena take part. We first review the basic equations of continuum mechanics within the scope of biological tissue. Based only on continuum mechanics and computational models as the ones described in this review, researchers have been able 


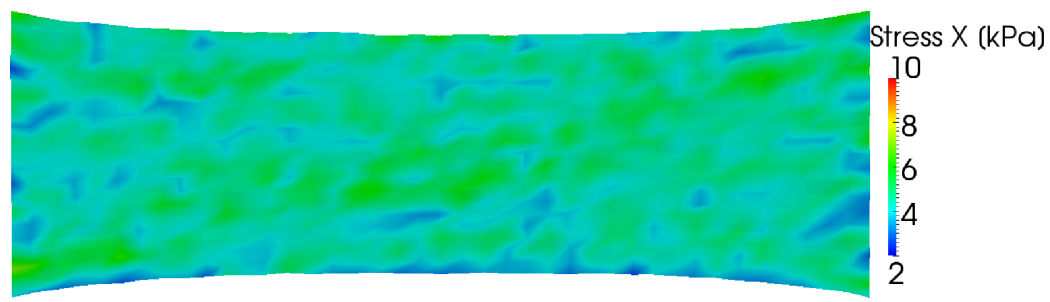

(a) Stress field in X direction at time step 10 .

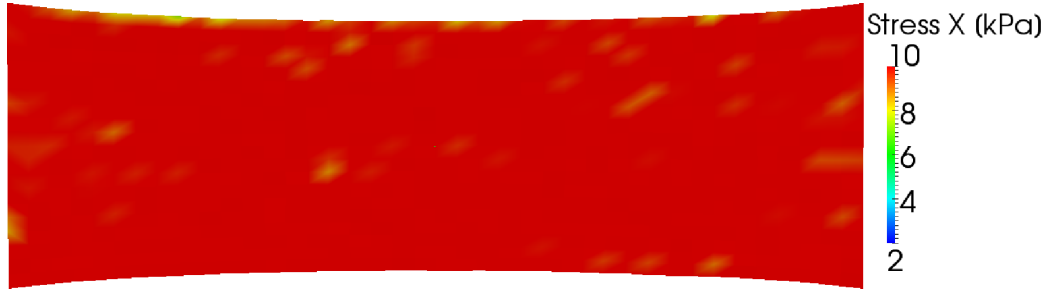

(b) Stress field in X direction at time step 100

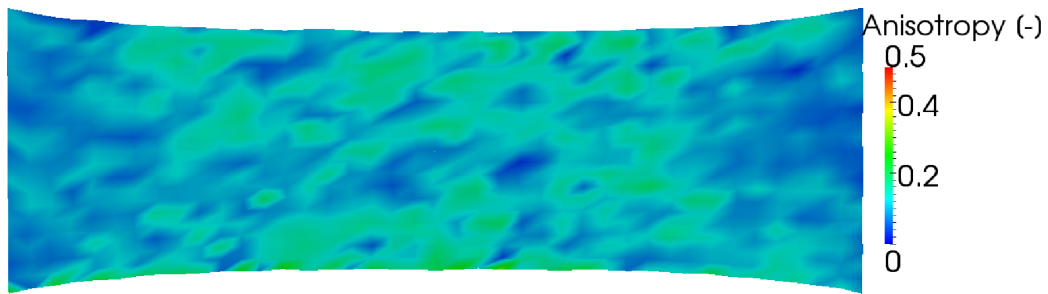

(c) Stress field in X direction at time step 10 .

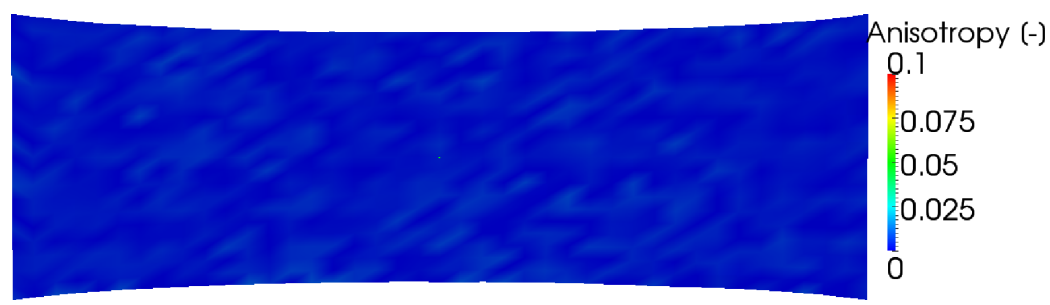

(d) Stress field in X direction at time step 100

Fig. 10: Evolution of stress for different boundary value problems, Dirichlet (a-b) and Neumann (c-d) for remodeling driven by strain (from ? with permission).

to model growth and remodeling in arteries (???), in the heart (?), bones (???), plants (?), sea shells (?). Researchers have been also investigating the evolution of tumors (??), wounds (??), and cells theoretically and computationally based in some of the approaches described in this review. 
There is not doubt that these continuum approaches have provided important results and have been able to give a clue about many aspects of the adaptation processes in biological tissue. The main advantages of all these continuum approaches is that the behavior of these macroscopically continuum-based models goes down to the definition of a SEDF that can completely define the mechanical behavior of the tissue.

Later, we also reviewed the finite element formulation given the continuum approach described herein. The finite element method have been widely, and basically the only one, used for the computational side of the problem. The numerical part of the problem has been focused, more than in the development of continuous Galerkin methods, on the coupling of different kind of phenomena and in the constitutive level of the problem. FEM have the advantages of relatively low time computing and a very well establish method to solve partial derivatives equations. Therefore the use of the finite element method has been established as the most straight forward numerical method to deal with the continuum approaches in biological tissues. Kuhl and co-workers, in series of theoretical and numerical problems stated some well defined description of the growth and remodeling in the context of open systems (??). The complexity of coupling different phenomena lies down on the correct description of the theoretical problem and and consistent linearization of the non-linear terms of the problem. Examples of consistent implicit formulation are e.g., the ones presented in ??.

7.2 Drawbacks in biological continuum models

One of the main drawbacks of growth adaptation processes, and particularly growth phenomena in large non homogeneous growth, is that they can not be computed in the context of a finite element models. Let's put as an example the growth of a tree from the very first seed, cellular growth or tissue development. The need of different techniques that makes any arbitrary growth 
feasible in terms of numerical point of view is, from our point of view, the most promising direction in the context of computational modeling of large growth processes in biological tissues.

In the constitutive level, numerical issues arise from complicated constitutive model adopted and the not trivial linearization of the strain energy function, see e.g. ??. They use quite a lot of phenomenological constitutive equation, describing the behavior at the micro-scale to reproduce the macro-scale behavior. In order to be able to reproduce in a continuum macroscopically sense, something that has its motivation in a very lower microscopical level, continuum models used to put in play a good amount of parameters. In some cases they are fitted to reproduce a given experimental result and other are just chosen to reproduce the phenomenon at hand with no much physical meaning. Therefore, the advantage of simplicity described above, also turns into a limitation. In this regard ? present a approach to describe the numerical computation of the consistent tangent moduli in finite inelasticity based o a perturbation of the deformation gradient. This provides a great advantage in the context of defining elastic tangent. Nevertheless, more biological microscopically motivated models are needed in order to feed macroscopically models of biological adaptation.

\subsection{Other perspectives}

Out of the continuum models described during this article there are other approaches followed in literature that deal with the growth remodeling and adaptation of biological tissue. For example, microstructural-based adaptation processes, agent-based and cell-centered models ? have been used for the description of a range of processes ??? where the components of the frame of study, e.g. cells, differentiate, proliferate and moves, giving as a result a new very different geometry than the original one. See e.g. Fig. ?? where the authors used agent-based and cell centered models to simulate the vasculogenesis process and the tissue growth around stent devices. 
Continuum models are a good option for describing macroscopically materials like the cardiovascular tissue and biochemical substances but cell-centered models can help to input experimental observations into continuum models. Agent-based models are based on the simulation of the actions and interactions of individual agents, gathered by individually and collectively effects. Agent-based models are made of "agents", with a particular heuristic and learning behavior. For example, an approach within the agent-based model is the cellular pots model (?). It is based on the postulation of a Hamiltonian function, based on the behavior of the agents, that is usually solved by Monte Carlo simulations (?).

As stated before, from the point of view of density growth defined as the creation, transport and interaction of biological substances over the tissue, the limitations lies down on the biological description of the problem and how numerically consistent and coupled one can make the numerical approach. And future lines of research will be focusing on interdisciplinary work within different disciplines (mathematics, engineering, biology, chemistry, etc) in order to accomplish more multiphysics multiscale approaches where each discipline look at a specific physic-scale of the problem. This is a path that all of us are seeing and looking after in the last years.

In terms of numerical point of view the treatment of large growth phenomena is more complicated. This is in the context of volumetric growth, in the sense that the morphology of the given body change abruptly. We have seen, for example, the case of vascologenesis, which could be seen as the growth from a more micro-scale point of view. There is, however, a gap between these two approaches that should be matched. One viable and convenient option is the use of meshless methods (??). Meshless methods lack of the mesh constraint by describing the behavior of the body of interest by a given point cloud. The basic idea is to approximate a given scalar function $u(\mathbf{x}, t)$ as $u(\mathbf{x}, t)=\sum_{i}^{n} \phi_{i}(\mathbf{x}) u_{i}(t)$, where $\phi_{i}(\mathbf{x})$ are the shape function and the $u_{i}(t)$ are the nodal values of the particle $i$ at $\mathbf{x} . i=1, \ldots, n$ is the set of nodes in the domain of interest. Although the approach is similar to FEM, here we use shape functions instead of interpolations, with the subsequent well know drawback in terms of imposing boundary conditions. A wide amount of shape 


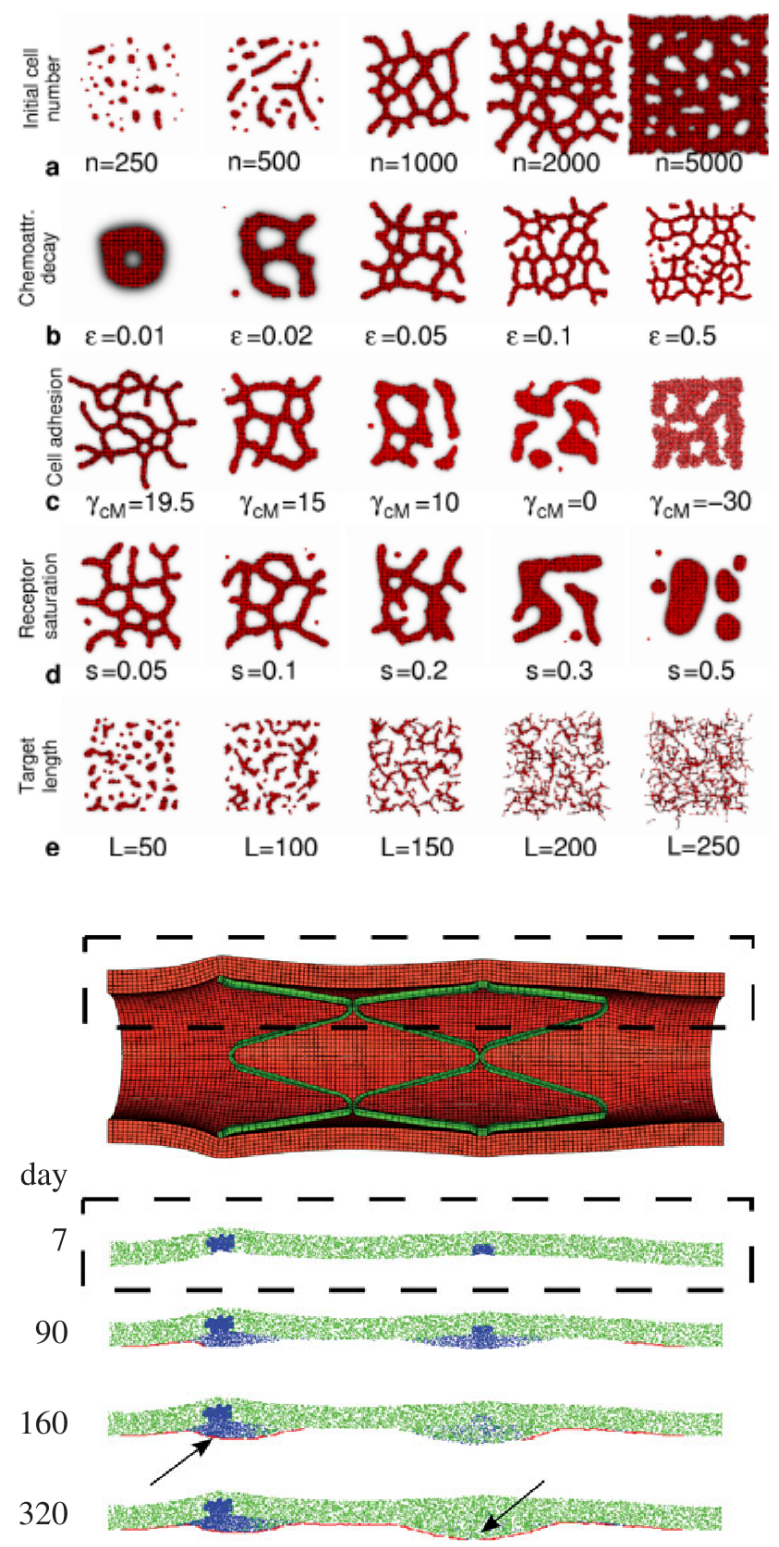

Fig. 11: Examples of growth processes in biology. Top, Hypothetical mechanisms of de novo vascular development ? (with permission). Bottom, ? showed the evolution of neointima due to the stresses induced in a artery due to stent placement, coupling both cell-centered model and FE models (reprinted from ?). 
function have been proposed over the years, beginning by smooth particles hydrodynamic (SPH), natural element method, Moving least squares and recently local-max entropy interpolants (??). Nevertheless, these Galerkin methods add a very promising path to integrate micro-scale models of cellular and particle interaction into large macroscale growth phenomena. Meshless methods have shown excellent capabilities to simulate extreme phenomena as many of the problems we face up to in biological adaptation.

\section{Conclusions}

In this article we have reviewed the continuum approach of adaptation processes in biological tissues. We particularly reviewed the last continuum models and numerical approaches on the effect of growth in terms of density and volume changes. We also looked at the effect of remodeling, meaning a change of the biological fibered structures, as the cardiovascular tissue or cells. We also discussed on the numerics of implementing adaptation constitutive laws within a finite element framework providing some algorithm recipes. All these theoretical and numerical techniques have been able to reproduce many of the biological adaptation processes that occurs in biological tissue. Researchers have modeled the thickening of arteries, heart expansion, wound healing, cell remodeling, etc. One of the more important drawback is related with the fact of dealing with such a complicated materials. Many assumptions and constitutive modeling have to be done in order to deal with them. Besides this issue, complications also arise when dealing with very large growth processes where classical numerical techniques, as the finite element methods, fail. Therefore, there are a necessity of integration of interdisciplinary efforts to bring together the features of continuum models with the more biological and micromechanical description of these processes. Also, integration of more flexible numerical techniques, e.g. meshless approaches, could help to go further in the study and simulation of many aspect of tissue adaptation (bone or brain development), cell reorganization processes (actin-myosin filaments, microtubular growth, cell shape remodeling, etc) and many oth- 
ers. We believe that integration of all these efforts will overcome many of these limitations along the future years.

P. Saez acknowledges support from the Spanish Ministry of Research and Innovation through the grant BES-009-028593 through which part of the research was conducted. PS also acknowledge Prof. Martinez and Prof. Pena for discussion and supervision. A special acknowledge also for Prof. Kuhl for many discussions and help during the last years.

\section{References}

V. Alastrue, B. Calvo, E. Pena, and M. Doblare. Biomechanical modeling of refractive corneal surgery. J Biomech Eng, 128(1):150-160, February 2006.

V. Alastrue, M. A. Martinez, M. Doblare, and A. Menzel. Anisotropic micro-sphere-based finite elasticity applied to blood vessel modelling. J Mech Phys Solids, 57(1):178-203, January 2009.

D. Ambrosi, G. A. Ateshian, E. M. Arruda, S. C. Cowin, J. Dumais, A. Goriely, G. A. Holzapfel, J. D. Humphrey, R. Kemkemer, E. Kuhl, J. E. Olberding, L. A. Taber, and K. Garikipati. Perspectives on biological growth and remodeling. J Mech Phys Solids, 59(4):863-883, April 2011.

A.R.A. Anderson and M.A.J. Chaplain. Continuous and discrete mathematical models of tumorinduced angiogenesis. 60(5):857-899-, 1998.

M. Arroyo and M. Ortiz. Local maximum-entropy approximation schemes: a seamless bridge between finite elements and meshfree methods. Int. J. Numer. Meth. Engng., 65(13):2167-2202, 2006.

G. A. Ateshian. On the theory of reactive mixtures for modeling biological growth. Biomech Model Mechan, 6(6):423-445, November 2007.

Gerard A Ateshian, Steve Maas, and Jeffrey A Weiss. Multiphasic finite element framework for modeling hydrated mixtures with multiple neutral and charged solutes. J Biomech Eng, 135(11): 
1110011-11100111, June 2013.

P. Bažant and B. H. Oh. Efficient numerical integration on the surface of a sphere. ZAMM-Z Angew Math Comput Me, 66(1):37-49, 1986.

P V Bayly, R J Okamoto, G Xu, Y Shi, and L A Taber. A cortical folding model incorporating stress-dependent growth explains gyral wavelengths and stress patterns in the developing brain. Phys Biol, 10(1):016005-, 2013.

P.V. Bayly, L.A. Taber, and C.D. Kroenke. Mechanical forces in cerebral cortical folding: A review of measurements and models. J Mech Behav Biomed Mater, 29(0):568-581, January 2014.

Z. P. Bazant and B. H. Oh. Microplane model for progressive fracture of concrete and rock. J Eng Mech-ASCE, 111(4):559-582, 1985.

Z. P. Bazant and P. C. Prat. Microplane model for brittle-plastic material .i. theory. J Eng MechASCE, 114(10):1672-1687, October 1988.

T. Belytschko, Y. Krongauz, D. Organ, M. Fleming, and P. Krysl. Meshless methods: An overview and recent developments. Comp Methods Appl M, 139(14):3-47, December 1996.

M. Ben Amar and A. Goriely. Growth and instability in elastic tissues. J Mech Phys Solids, 53(10): 2284-2319, October 2005.

Eric Bonabeau. Agent-based modeling: methods and techniques for simulating human systems. Proc Natl Acad Sci U S A, 99 Suppl 3:7280-7287, May 2002.

C. J. Boyle, A. B. Lennon, M. Early, D. J. Kelly, C. Lally, and P. J. Prendergast. Computational simulation methodologies for mechanobiological modelling: a cell-centred approach to neointima development in stents. Philos T Roy Soc A, 368(1921):2919-2935, June 2010.

Silvia Budday, Charles Raybaud, and Ellen Kuhl. A mechanical model predicts morphological abnormalities in the developing human brain. Sci Rep, 4:5644, 2014a.

Silvia Budday, Paul Steinmann, and Ellen Kuhl. The role of mechanics during brain development. J Mech Phys Solids, 72:75-92, Dec 2014b. 
Adrian Buganza-Tepole and Ellen Kuhl. Systems-based approaches toward wound healing. Pediatr Res, 73(4-2):553-563, April 2013.

Adrian Buganza-Tepole and Ellen Kuhl. Computational modeling of chemo-bio-mechanical coupling: a systems-biology approach toward wound healing. doi: 10.1080/10255842.2014.980821.

Ingo Burgert and Peter Fratzl. Mechanics of the expanding cell wall. In Jean-Pierre Verbelen and Kris Vissenberg, editors, Plant Cell Monographs, volume 6, pages 191-215-. Springer Berlin Heidelberg, 2007.

I. Carol, M. Jirasek, and Z. Bazant. A thermodynamically consistent approach to microplane theory. part i. free energy and consistent microplane stresses. Int J Solids Struct, 38(17):2921-2931, April 2001.

Jhih-Wei Chu and Gregory A. Voth. Allostery of actin filaments: Molecular dynamics simulations and coarse-grained analysis. Proc Nat Acad Sci USA, 102(37):13111-13116, September 2005.

S.C. Cowin and D.H. Hegedus. Bone remodeling i: theory of adaptive elasticity. 6(3):313-326-, 1976.

G. H. Dai, M. R. Kaazempur-Mofrad, S. Natarajan, Y. Z. Zhang, S. Vaughn, B. R. Blackman, R. D. Kamm, G. Garcia-Cardena, and M. A. Gimbrone. Distinct endothelial phenotypes evoked by arterial waveforms derived from atherosclerosis-susceptible and -resistant regions of human vasculature. Proc Nat Acad Sci USA, 101(41):14871-14876, October 2004.

R. De and S. A. Safran. Dynamical theory of active cellular response to external stress. Phys Rev E, 78(3):031923, September 2008.

R. De, A. Zemel, and S. A. Safran. Dynamics of cell orientation. Nat Phys, 3(9):655-659, September 2007.

W. M. Deen. Analysis of Transport Phenomena. Oxford University Press, 2011.

D. E. Discher, P. Janmey, and Y. L. Wang. Tissue cells feel and respond to the stiffness of their substrate. Science, 310(5751):1139-1143, November 2005. 
N. J. B. Driessen, G. W. M. Peters, J. M. Huyghe, C. V. C. Bouten, and F. P. T. Baaijens. Remodelling of continuously distributed collagen fibres in soft connective tissues. J Biomech, 36 (8):1151-1158, August 2003.

N. J. B. Driessen, M. A. J. Cox, C. V. C. Bouten, and F. P. T. Baaijens. Remodelling of the angular collagen fiber distribution in cardiovascular tissues. Biomech Model Mechan, 7(2):93-103, April 2008.

Benjamin S. Elkin, Mohammed A. Shaik, and Barclay Morrison. Fixed negative charge and the donnan effect: adescription of the driving forces associated with brain tissue swelling andoedema. Philos T Roy Soc A, 368(1912):585-603, January 2010.

Marcelo Epstein and Gerard A. Maugin. Thermomechanics of volumetric growth in uniform bodies. Int J Plasticity, 16(78):951-978, June 2000.

C. A. Figueroa, S. Baek, C. A. Taylor, and J. D. Humphrey. A computational framework for fluid-solid-growth modeling in cardiovascular simulations. Comp Methods Appl M, 198(45-46): 3583-3602, 2009.

H Fountain, 2012. URL http://www.nytimes.com/.

Y. C. Fung. Biomechanics: Mechanical Properties of Living Tissues. Springer, 1990.

YC Fung and SQ Liu. Change of residual strains in arteries due to hypertrophy caused by aortic constriction. Circ Res, 65(5):1340-1349, 1989.

J. F. Ganghoffer. Mechanical modeling of growth considering domain variation. part ii: Volumetric and surface growth involving eshelby tensors. J Mech Phys Solids, 58(9):1434-1459, September 2010.

T. C. Gasser, R. W. Ogden, and G. A. Holzapfel. Hyperelastic modelling of arterial layers with distributed collagen fibre orientations. J Roy Soc Interface, 3:15-35, 2006.

R. L. Gleason and J. D. Humphrey. A mixture model of arterial growth and remodeling in hypertension: Altered muscle tone and tissue turnover. J Vasc Res, 41(4):352-363, 2004. 
R.L. Gleason and J.D. Humphrey. Effects of a sustained extension on arterial growth and remodeling: a theoretical study. J Biomech, 38(6):1255-1261, June 2005.

S. Goektepe, O. J. Abilez, and E. Kuhl. A generic approach towards finite growth with examples of athlete's heart, cardiac dilation, and cardiac wall thickening. J Mech Phys Solids, 58(10): 1661-1680, October 2010

Xiaomei Guo and Ghassan S. Kassab. Variation of mechanical properties along the length of the aorta in c57bl/6 mice. American Journal of Physiology - Heart and Circulatory Physiology, 285(6): H2614-H2622, November 2003.

F. Guilak and V.C. Mow The mechanical environment of the chondrocyte: a biphasic finite element model of cell-matrix interactions in articular cartilage. J Biomech, 33(12):16331673, December 2000.

S. Goektepe, O. J. Abilez, and E. Kuhl. A generic approach towards finite growth with examples of athlete's heart, cardiac dilation, and cardiac wall thickening. Journal of the Mechanics and Physics of Solids, 58(10):1661-1680, October 2010.

Serdar Goektepe, A. Menzel, and E. Kuhl. The generalized hill model: A kinematic approach towards active muscle contraction. J Mech Phys Solids, 72(0):20-39, December 2014

T. P. Harrigan and J. J. Hamilton. An analytical and numerical study of the stability of bone remodeling theories - dependence on microstructural stimulus. J Biomech, 25(5):477-488, May 1992.

E. A. Heidenreich, J. M. Ferrero, M. Doblare, and J. F. Rodriguez. Adaptive macro finite elements for the numerical solution of monodomain equations in cardiac electrophysiology. Ann Biomed Eng, 38(7):2331-2345, July 2010.

G. Himpel, E. Kuhl, A. Menzel, and P. Steinmann. Computational modelling of isotropic multiplicative growth. CMES, 8(2):119-134, 2005.

G. Himpel, A. Menzel, E. Kuhl, and P. Steinmann. Time-dependent fibre reorientation of transversely isotropic continua . finite element formulation and consistent linearization. Intl J Numer 
Meth Eng, 73(10):1413-1433, March 2008.

G. A. Holzapfel. Encyclopedia of Computational Mechanics. 2004.

G. A. Holzapfel, T. C. Gasser, and R. W. Ogden. A new constitutive framework for arterial wall mechanics and a comparative study of material models. J Elasticity, 61(1):1-48, July 2000.

J. D. Humphrey. Mechanics of the arterial wall: Review and directions. Crit. Rev. Bio. Eng., 23 (1-2):1-162, 1995 .

J. D. Humphrey. Continuum biomechanics of soft biological tissues. Proceedings of the Royal Society A-mathematical Physical and Engineering Sciences, 459(2029):3-46, January 2003.

J. D. Humphrey. Need for a continuum biochemomechanical theory of soft tissue and cellular growth and remodeling. In Biomechanical Modelling at the Molecular, Cellular and Tissue Levels. Springer Vienna, 2009.

J. D. Humphrey and S. Na. Elastodynamics and arterial wall stress. Ann Biomed Eng, 30(4): 509-523, April 2002.

J. D. Humphrey and K. R. Rajagopal. A constrained mixture model for growth and remodeling of soft tissues. Math Models Methods Appl Sci, 12(3):407-430, March 2002.

J.D. Humphrey and K.R. Rajagopal. A constrained mixture model for arterial adaptations to a sustained step change in blood flow. Biomech Model Mechan, V2(2):109-126, November 2003.

C. Hurschler, B. Loitz-Ramage, and R. Vandervir Jr. A structurally based stress-stretch relationship for tendon and ligament. J Biomech Eng-T Asme, 119:392-399, 1997.

J.E. Hurtado and A.H. Barbat. Monte carlo techniques in computational stochastic mechanics. 5 (1):3-29-, 1998. ISSN 1134-3060.

S. Imatani and G. A. Maugin. A constitutive model for material growth and its application to three-dimensional finite element analysis. Mech Res Commun, 29(6):477-483, 2002.

D. E. Ingber. Tensegrity i. cell structure and hierarchical systems biology. J Cell Sci, 116(7): 1157-1173, April 2003. 
Yi Jiang, Jelena Pjesivac-Grbovic, Charles Cantrell, and James P Freyer. A multiscale model for avascular tumor growth. Biophysical Journal, 89(6):3884-3894, September 2005.

D. Kardas, U. Nackenhorst, and D. Balzani. Computational model for the cell-mechanical response of the osteocyte cytoskeleton based on self-stabilizing tensegrity structures. Biomech Model Mechan, 2012.

I. Karsaj, C. Sansour, and J. Soric. The modelling of fibre reorientation in soft tissue. Biomech Model Mechan, 8(5):359-370, October 2009.

Taeyoon Kim, Wonmuk Hwang, Hyungsuk Lee, and Roger D Kamm. Computational analysis of viscoelastic properties of crosslinked actin networks. PLoS Comput Biol, 5(7):e1000439, Jul 2009.

Yangjin Kim, Magdalena A. Stolarska, and Hans G. Othmer. The role of the microenvironment in tumor growth and invasion. Progress in Biophysics and Molecular Biology, 106(2):353-379, August 2011.

Doron Klepach, Lik Chuan Lee, Jonathan F Wenk, Mark B Ratcliffe, Tarek I Zohdi, Jose A Navia, Ghassan S Kassab, Ellen Kuhl, and Julius M Guccione. Growth and remodeling of the left ventricle: A case study of myocardial infarction and surgical ventricular restoration. Mech Res Commun, 42:134-141, Jun 2012.

S. M. Klisch, R. L. Sah, and A. Hoger. A cartilage growth mixture model for infinitesimal strains: solutions of boundary-value problems related to in vitro growth experiments. Biomech Model Mechan, 3(4):209-223, June 2005.

O. Kratky and G. Porod. Rontgenuntersuchung geloster fadenmolekule. Recl Trav Cnim Pay B, 68 (12):1106-1122, 1949.

E. Kuhl. Theory and numerics of open system continuum thermodynamics - spatial and material settings -. PhD thesis, University of Kaiserslautern, 2003.

E. Kuhl and P. Steinmann. Theory and numerics of geometrically non-linear open system mechanics. Int J Numer Meth Eng, 58(11):1593-1615, 2003. 
E. Kuhl, P. Steinmann, and I. Carol. A thermodynamically consistent approach to microplane theory. part ii. dissipation and inelastic constitutive modeling. Int J Solids Struct, 38(17):29332952, April 2001.

E. Kuhl, A. Menzel, and P. Steinmann. Computational modeling of growth - a critical review, a classification of concepts and two new consistent approaches. Comput Mech, 32(1-2):71-88, September 2003.

E. Kuhl, K. Garikipati, E. M. Arruda, and K. Grosh. Remodeling of biological tissue: Mechanically induced reorientation of a transversely isotropic chain network. J Mech Phys Solids, 53(7):15521573, July 2005.

W. M. Lai, J. S. Hou and V. C. Mow A Triphasic Theory for the Swelling and Deformation Behaviors of Articular Cartilage J Biomech Eng-T Asme, 113(3):245258\&, 1991.

E. H. Lee. Elastic-plastic deformation at finite strains. Journal of Applied Mechanics, 36(1):1-\&, 1969.

L. C. Lee, M. Genet, G. Acevedo-Bolton, K. Ordovas, J. M. Guccione, and E. Kuhl. A computational model that predicts reverse growth in response to mechanical unloading. Biomech Model Mechanobiol, Jun 2014.

V. A. Lubarda and A. Hoger. On the mechanics of solids with a growing mass. Int J Solids Struct, 39(18):4627-4664, September 2002.

J. E. Marsden and T. J. R. Hughes. Mathematical Foundations of Elasticity. Dover Publications, 1994.

J. E. Marsden and T. S. Ratiu. Introduction to Mechanics and Symmetry: A Basic Exposition of Classical Mechanical Systems. Springer Verlag, 1999.

B. Maurin, P. Canadas, H. Baudriller, P. Montcourrier, and N. Bettache. Mechanical model of cytoskeleton structuration during cell adhesion and spreading. J Biomech, 41(9):2036-2041, 2008.

A. McCulloch, J. Bassingthwaighte, P. Hunter, and D. Noble. Computational biology of the heart: from structure to function. Prog Biophys Mol Biol, 69(2-3):153-155, 1998. 
A. Menzel. Modelling of anisotropic growth in biological tissues. Biomech Model Mechan, 3(3): 147-171, March 2004.

A. Menzel. A fibre reorientation model for orthotropic multiplicative growth. Biomech Model Mechan, 6:303-320, 2007.

A. Menzel and T. Waffenschmidt. A microsphere-based remodelling formulation for anisotropic biological tissues. Phil Trans R Soc A, 367(1902):3499-3523, September 2009.

Roeland M H Merks and James A Glazier. Dynamic mechanisms of blood vessel growth. Nonlinearity, 19(1):C1-, 2006.

Roeland M.H. Merks and James A. Glazier. A cell-centered approach to developmental biology. Physica A: Statistical Mechanics and its Applications, 352(1):113-130, July 2005.

C. Miehe, S. Göktepe, and F. Lulei. A micro-macro approach to rubber-like materials-part i: the non-affine micro-sphere model of rubber elasticity. J Mech Phys Solids, 52(11):2617-2660, November 2004.

Christian Miehe. Numerical computation of algorithmic (consistent) tangent moduli in large-strain computational inelasticity. Computer Methods in Applied Mechanics and Engineering, 134(34):223240, August 1996.

M.R. K. Mofrad and R D. Kamm. Cytoskeletal Mechanics - Models and Measurements. Cambridge University Press, 2006.

D.E. Moulton, A. Goriely, and R. Chirat. Mechanical growth and morphogenesis of seashells. J Theor Biol, 311(0):69-79, October 2012.

S. I. Murtada, M. Kroon, and G. A. Holzapfel. A calcium-driven mechanochemical model for prediction of force generation in smooth muscle. Biomech Model Mechan, 9(6):749-762, December 2010.

A. N. Natali, P. G. Pavan, E. L. Carniel, M. E. Lucisano, and G. Taglialavoro. Anisotropic elastodamage constitutive model for the biomechanical analysis of tendons. Med. Eng. Phys., 27(3): 209-214, April 2005. 
Vinh Phu Nguyen, Timon Rabczuk, Stephane Bordas, and Marc Duflot. Meshless methods: A review and computer implementation aspects. Math Comput Simulat, 79(3):763-813, December 2008. .

R.W. Ogden. Nonlinear elasticity, anisotropy, material stability and residual stresses in soft tissues. In G.A. Holzapfel and R.W. Ogden, editors, Biomechanics of Soft Tissue in Cardiovascular Systems, number 441 in CISM Courses and Lectures, pages 65-108. Springer, 2003.

G. K. Owens, P. S. Rabinovitch, and S. M. Schwartz. Smooth-muscle cell hypertrophy versus hyperplasia in hypertension. P Natl Acad Sci-biol, 78(12):7759-7763, 1981.

A. Pandolfi and F. Manganiello. A model for the human cornea: constitutive formulation and numerical analysis. Biomech Model Mechan, 5(4):237-246, November 2006.

E. Peña, B. Calvo, M. A. Martinez, and M. Doblare. A three-dimensional finite element analysis of the combined behavior of ligaments and menisci in the healthy human knee joint. J Biomech, 39(9):1686-1701, 2006.

M.K. Rausch, A. Dam, S. Göktepe, O.J. Abilez, and E. Kuhl. Computational modeling of growth: Systemic and pulmonary hypertension in the heart. Biomech Model Mechanobiol, 10:799-811, 2011.

J. C. Rice, S. C. COWIN, and J. A. BOWMAN. On the dependence of the elasticity and strength of cancellous bone on apparent density. J Biomech, 21(2):155-168, 1988.

E. K. Rodriguez, A. Hoger, and A. D. McCulloch. Stress-dependent finite growth in soft elastic tissues. J Biomech, 27(4):455-467, April 1994.

P. Saez, E. Pena, M.A. Martinez, and E. Kuhl. Mathematical modeling of collagen turnover in biological tissue. J Math Bio., 2012.

R. Skalak. Growth as a finite displacement field. In D. E. Carlson and R. T. Shields, editors, IUTAM Symposium on Finite Elasticity, pages 348-355. Martinus Nijhoff, 1982.

R. Skalak, G. Dasgupta, M. Moss, E. Otten, P. Dullemeijer, and H. Vilmann. Analytical description of growth. J Theor Biol, 94(3):555-577, February 1982. 
P. Steinmann. Formulation and computation of geometrically non-linear gradient damage. Intl J Numer Meth Eng, 46(5):757-779, October 1999.

N. Sukumar. Construction of polygonal interpolants: a maximum entropy approach. Int. J. Numer. Meth. Engng., 61(12):2159-2181, 2004.

P. Saez, E. Pena, M. Doblare, and M.A. Martinez. Hierarchical micro-adaptation of biological structures by mechanical stimuli. Int J Solids Struct, 50(1415):2353-2370, July 2013a.

P. Saez, E. Pena, and M.A. Martinez. A structural approach including the behavior of collagen cross-links to model patient-specific human carotid arteries. Ann Biomed Eng, 42(6):1158-1169, 2014a.

P. Saez, E. Pena, M. A. Martinez, and E. Kuhl. Computational modeling of hypertensive growth in the human carotid artery. Comput Mech, 53(6):1183-1196, Jun 2014b.

P. Saez, E. Pena, J. M. Tarbell, M. A. Martinez. Computational model of collagen turnover in carotid arteries during hypertension. Submitted for publication.

L. A. Taber. Biomechanics of growth, remodeling, and morphogenesis. Appl. Mech. Rev., 48:487-545, 1995.

Larry A. Taber and Daniel W. Eggers. Theoretical study of stress-modulated growth in the aorta. J Theor Biol, 180(4):343-357, June 1996.

A. Buganza Tepole, C. J. Ploch, J. Wong, A. K Gosain, and E. Kuhl. Growing skin: A computational model for skin expansion in reconstructive surgery. J Mech Phys Solids, 59(10):2177-2190, Oct 2011.

A. Valentin and J. D. Humphrey. Evaluation of fundamental hypotheses underlying constrained mixture models of arterial growth and remodelling. P Roy Soc Lond A Mat, 367(1902):3585-3606, September 2009

C. Valero, E. Javierre, J. M. Garcia-Aznar, and M. Jose Gmez-Benito. A cell-regulatory mechanism involving feedback between contraction and tissue formation guides wound healing progression. PLoS One, 9(3):e92774, 2014. 
T. Waffenschmidt, A. Menzel, and E. Kuhl. Anisotropic density growth of bone-a computational micro-sphere approach. Int J Solids Struct, 49(14):1928-1946, July 2012.

H. Weinans, R. Huiskes, and H.J. Grootenboer. The behavior of adaptive bone-remodeling simulation models. J Biomech, 25(12):1425-1441, December 1992.

S. Weinbaum, S. C. Cowin, and Y. Zeng. A model for the excitation of osteocytes by mechanical loading-induced bone fluid shear stresses. J Biomech, 27(3):339-360, March 1994.

J. Welty, C. E. Wicks, G. L. Rorrer, and R. E. Wilson. Fundamentals of Momentum, Heat and Mass Transfer. John Wiley \& Sons, 2008.

J. Wong, S. Goktepe, and E. Kuhl. Computational modeling of electrochemical coupling: A novel finite element approach towards ionic models for cardiac electrophysiology. Comput Method Appl M, 200(45-46):3139-3158, 2011.

A. M Zoellner, M. A Holland, K. S Honda, A. K Gosain, and E. Kuhl. Growth on demand: reviewing the mechanobiology of stretched skin. J Mech Behav Biomed Mater, 28:495-509, Dec 2013. 